\title{
Persistent competition models on two complementary nutrients with density-dependent consumption rates
}

\author{
Fethi Borsali $^{1}$ - Karim Yadi ${ }^{1}$
}

Received: 2 February 2018 / Accepted: 5 May 2018 / Published online: 15 May 2018

(C) Fondazione Annali di Matematica Pura ed Applicata and Springer-Verlag GmbH Germany, part of Springer Nature 2018

\begin{abstract}
We give a result of uniform persistence for a theoretical competition model of 3 species of microorganisms on 2 complementary nutrients in a chemostat with densitydependent growth functions. The proof consists of giving first sufficient conditions of persistence, which is by the way available for more than 3 species. Then, in the case of 2 species, we show under additional assumptions, and with the use of the so-called Hofbauer-Hutson theorem, that it is possible to obtain a uniform persistence around a positive equilibrium point. Finally, the main result is established from what preceeds and by means of Thieme-Zhao theorem. These results are illustrated by some numerical simulations.
\end{abstract}

Keywords Dynamical systems · Global stability · Uniform persistence - Chemostat · Complementary nutrients · Density dependence

Mathematics Subject Classifications $34 \mathrm{C} 11 \cdot 37$ N25 $\cdot 92 \mathrm{~B} 05$

\section{Introduction}

The chemostat is a laboratory apparatus used for continuous cultivation of microorganisms in which the effect of limiting nutrient on the population of microorganisms can be investigated. A theoretical approach to the description of the competition of two or more populations of microorganisms on a limiting nutrient in a chemostat has been developed in the book of Smith and Waltman [18] (see also Harmand et al. [8], Ajbar and Alhumaizi [1] and the references therein). Our interest goes to models where growth functions are obtained by the combination of two limiting nutrients. The different classifications of limiting nutrients were

\footnotetext{
$凶 \quad$ Fethi Borsali

fethi_borsali@yahoo.com

Karim Yadi

k_yadi@mail.univ-tlemcen.dz

1 Laboratoire Systèmes Dynamiques et Applications, University of Tlemcen, BP 119, 13000 Tlemcen, Algeria
} 
introduced by Rapport [17] and Covich [6]. We mainly distinguish between two types, the socalled complementary nutrients, that is nutrients of different essential substances which are independently required for growth, and substitutable nutrients, that is alternative sources of an essential substance. Note that the first type is called complementary by Leon and Tumpson [12] and essential by Tilman [20]. Leon and Tumpson found the conditions of local stability of a positive equilibrium point and hence conditions of coexistence of the two populations. Hsu et al. [10] made a global study when the growth functions are of Michaelis-Menten type and obtained the classical results of the two dimensional Lotka-Volterra competition model. The same model with general monotonic and non-monotonic growth functions was studied by Butler and Wolkowicz [5]. Li [13] examined a model generalizing that of Butler and Wolkowicz with several populations of microorganisms and for a class of general growth functions. He proved global stability of a positive equilibrium point under some conditions. Mazenc and Malisoff [16] considered a more general model with $M$ resources and $N$ species and different removal rates. When $M=N$, they obtained a conditional existence of a positive equilibrium point by Brouwer degree theory. For particular families of the growth functions they proved the global asymptotic stability of such a point with the use of Lyapunov functions. Recently, Borsali and Yadi [4] studied the same model of two microorganisms where they added terms of interspecific interaction. They proved the permanence of the model and the existence of a positive equilibrium point under some conditions. On the other hand, many models where the growth function depends not only on the substrate but also on the microorganism, have been considered. In this context, Arditi and Ginzburg [2,3] developed predator-prey models with functional responses depending on the prey and the predator. They suggested a class of functional responses which take into account the interference between the predators and depending on the amount of prey consumed by one predator that is on the ratio of the amount of consumed prey and the predator per unit time : the consumption rate decreases proportionally with the abundance of predators. The models with this type of functional response are called ratio-dependent. This approach has also been applied in competitive models in the chemostat. More exactly, in the following chemostat model of competition of $n$ species " $x_{i}$ " on a single nutrient " $s$ ",

$$
\begin{aligned}
& \dot{s}=\left(s^{\circ}-s\right) D-\sum_{i=1}^{n} \frac{1}{y_{i}} \mu_{i}(s, x) x_{i}, \\
& \dot{x}_{i}=\left(\mu_{i}(s, x)-D\right) x_{i}, \quad i \in\{1,2, \ldots, n\},
\end{aligned}
$$

the growth function, denoted by $\mu_{i}(s, x)$, depends on the substrate and microorganisms. These models are rather called density-dependent.

Lobry and Mazenc [15] examined the following general density-dependent competition model called intra-specific competition model :

$$
\begin{aligned}
\dot{s} & =f(s)-\sum_{i=1}^{n} \frac{1}{y_{i}} \mu_{i}\left(s, x_{i}\right) x_{i}, \\
\dot{x}_{i} & =\left(\mu_{i}\left(s, x_{i}\right)-d_{i}\right) x_{i}, \quad i \in\{1,2, \ldots, n\} .
\end{aligned}
$$

Here, $x_{i}$ is the biomass of the $i^{t h}$ population in the chemostat, $s$ is the concentration of the nutrient, $s^{\circ}$ is the input concentration of the resource $s, D$ is the dilution rate, $y_{i}$ is the growth yield constant, and $d_{i}$ is the removal rate of the $i^{t h}$ species. The function $f$ is a positive function and of class $C^{1}$. The growth function $\mu_{i}$ is supposed to vanish at $s=0$, to be increasing with respect to $s$ and decreasing with respect to $x_{i}$. This assumption is natural. It ensures that the growth function is zero in the absence of the nutrient, increases with 
the amount of substrate, and decreases with the abundance of the microorganism. Under some assumptions, they showed that the model (2) has an interior equilibrium point globally asymptotically stable and therefore the coexistence of all the species of microorganisms. The demonstration is based on the construction of a Lyapunov function. Another study of the delay density-dependent model with the growth yield constants depending on the substrate was conducted by Xu and Li [14]. They also have the same result as that of Lobry and Mazenc [15].

The objective of the present paper is to study a density-dependent competition model of several microorganisms on two complementary nutrients. The model is written :

$$
\begin{aligned}
\dot{s} & =\left(s^{\circ}-s\right) D-\sum_{i=1}^{n} \frac{1}{y_{s i}} \mu_{i}\left(s, r, x_{i}\right) x_{i}, \\
\dot{r} & =\left(r^{\circ}-r\right) D-\sum_{i=1}^{n} \frac{1}{y_{r i}} \mu_{i}\left(s, r, x_{i}\right) x_{i}, \\
\dot{x}_{i} & =\left(\mu_{i}\left(s, r, x_{i}\right)-D\right) x_{i}, \quad i \in\{1,2, \ldots, n\},
\end{aligned}
$$

where the functions $\mu_{i}\left(s, r, x_{i}\right):=\min \left(p_{i}\left(s, x_{i}\right), q_{i}\left(r, x_{i}\right)\right)$ satisfy the following assumptions :

H1. The functions $s \mapsto p_{i}\left(s, x_{i}\right)$ and $r \mapsto q_{i}\left(r, x_{i}\right)$ are positive, continuously differentiable, increasing and such that $p_{i}\left(0, x_{i}\right)=q_{i}\left(0, x_{i}\right)=0$.

H2. The functions $x_{i} \mapsto p_{i}\left(s, x_{i}\right)$ and $x_{i} \mapsto q_{i}\left(r, x_{i}\right)$ are positive, continuously differentiable and decreasing.

Since $p_{i}$ and $q_{i}$ are $C^{1}$, the functions $\mu_{i}$ are locally Lipschitz. In particular, the assumptions $\mathrm{H} 1$ and $\mathrm{H} 2$ insure the existence and uniqueness property of solutions of (3) for prescribed initial conditions in $\mathbb{R}_{+}^{n+2}$. Moreover, the break-even concentrations will be defined as being the respective values $\lambda_{s_{i}}$ and $\lambda_{r_{i}}$ of $s$ and $r$ such that $p_{i}\left(\lambda_{s_{i}}, 0\right)=q_{i}\left(\lambda_{r_{i}}, 0\right)=D$.

This work should be seen as a contribution to the mathematical study of theoretical chemostat models in at least two aspects. The first one lies in the framework of multinutrients models, for which the references are less abundant. The second one concerns the densitydependence notion for which we believe that it will lead to new interesting questions. Even if this article in inspired by works in [15], the mathematical treatment turns to be more complex, except perhaps for the persistence result of Theorem 2.5 bellow. The aim of this work being theoretical, we do not give the connection with any realistic biological experiment.

In Sect. 2, we first show that the positive solutions of (3) are positively bounded and give conditions of washing out. We then prove the persistence of the model under some assumptions (Theorem 2.5). Section 3 is devoted to the study of the same model limited to two microorganisms and for which we obtain a uniform persistence result under some assumptions (Theorem 3.3). Moreover, we deduce that there exists a positive equilibrium point which is globally asymptotically stable (Theorem 3.4). Finally, in Sect. 4, thanks to the previous results we prove that our model (3) with three microorganisms is actually uniformly persistent and that there exists at least a positive equilibrium point (Theorem 4.1). Some numerical simulations are presented in this article to illustrate the results. Note that one can make the conjecture that the equilibrium point for the last case is unique and attractive. 


\section{Persistence in case of several species of microorganisms}

\subsection{Elementary properties}

The first two propositions recall the usual properties of dissipativity (i.e. the solutions are positively bounded), invariance and conditions of washing out. The proof of the first proposition is classical and is omitted.

Proposition 2.1 Under assumptions $H 1$ and H2, the system (3) is dissipative and the positive orthant $\mathbb{R}_{+}^{n+2}$ is positively invariant. More exactly, there exists $t_{0}>0$ such for all $t>t_{0}$ one has $s(t)<s^{\circ}, r(t)<r^{\circ}$ and $x_{i}(t)<\min \left(y_{s i} s^{\circ}, y_{r i} r^{\circ}\right), i \in\{1,2, \ldots, n\}$.

Proposition 2.2 Suppose that H1 and H2 are satisfied. If $s^{\circ}<\lambda_{s i}$ or $r^{\circ}<\lambda_{\text {ri }}$ then, for all positive initial conditions, one has

$$
\lim _{t \rightarrow+\infty} s(t)=s^{\circ}, \lim _{t \rightarrow+\infty} r(t)=r^{\circ} \text { and } \lim _{t \rightarrow+\infty} x_{i}(t)=0 .
$$

Proof If $s^{\circ}<\lambda_{s i}$ or $r^{\circ}<\lambda_{\text {ri }}$ for all $i \in\{1,2, \ldots, n\}$, then there exists a real constant $\varepsilon>0$ such that $s^{\circ}+\varepsilon<\lambda_{s i}$ or $r^{\circ}+\varepsilon<\lambda_{r i}$.

From Proposition 2.1, we deduce that

$$
\exists t_{0}>0: \forall t>t_{0} s(t)<s^{\circ}+\varepsilon, r(t)<r^{\circ}+\varepsilon .
$$

On the other hand, from the constant variational formula, we have :

$$
\begin{aligned}
x_{i}(t)= & x_{i}(0) \exp \left(\int_{0}^{t}\left(\mu_{i}\left(s(\tau), r(\tau), x_{i}(\tau)\right)-D\right) \mathrm{d} \tau\right), \\
= & x_{i}(0) \exp \left[\int_{0}^{t_{0}}\left(\mu_{i}\left(s(\tau), r(\tau), x_{i}(\tau)\right)-D\right) \mathrm{d} \tau\right. \\
& \left.+\int_{t_{0}}^{t}\left(\mu_{i}\left(s(\tau), r(\tau), x_{i}(\tau)\right)-D\right) \mathrm{d} \tau\right] .
\end{aligned}
$$

Hence,

$$
x_{i}(t)=C x_{i}(0) \exp \left(\int_{t_{0}}^{t}\left(\mu_{i}\left(s(\tau), r(\tau), x_{i}(\tau)\right)-D\right) \mathrm{d} \tau\right),
$$

where

$$
C:=\exp \left(\int_{0}^{t_{0}}\left(\mu_{i}\left(s(\tau), r(\tau), x_{i}(\tau)\right)-D\right) \mathrm{d} \tau\right)>0 .
$$

By assumption H2, we obtain :

$$
\begin{aligned}
x_{i}(t) & <C x_{i}(0) \exp \left(\int_{t_{0}}^{t}\left(\left(\mu_{i}\left(s^{\circ}+\varepsilon, r^{\circ}+\varepsilon, x_{i}(\tau)\right)-D\right)\right) \mathrm{d} \tau\right) \\
& <C x_{i}(0) \exp \left(\int_{t_{0}}^{t}\left(\left(\mu_{i}\left(s^{\circ}+\varepsilon, r^{\circ}+\varepsilon, 0\right)-D\right)\right) \mathrm{d} \tau\right) .
\end{aligned}
$$

Finally, we have :

$$
x_{i}(t)<C x_{i}(0) \exp \left(A_{i}\left(t-t_{0}\right)\right),
$$


where $A_{i}:=\mu_{i}\left(s^{\circ}+\varepsilon, r^{\circ}+\varepsilon, 0\right)-D$. The real constant $A_{i}$ is negative since $s^{\circ}+\varepsilon<\lambda_{s i}$ or $r^{\circ}+\varepsilon<\lambda_{r i}$ for all $i \in\{1,2, \ldots, n\}$. Finally, the solutions of the model (with positive initial conditions) are positive, then :

$$
\lim _{t \rightarrow+\infty} x_{i}(t)=0
$$

Now, let us set

$$
\begin{aligned}
& \Sigma_{1}:=s+\sum_{i=1}^{n} \frac{1}{y_{s i}} x_{i}, \\
& \Sigma_{2}:=r+\sum_{i=1}^{n} \frac{1}{y_{r i}} x_{i},
\end{aligned}
$$

which transforms the system (3) in :

$$
\begin{aligned}
& \dot{\Sigma}_{1}=D\left(s^{\circ}-\Sigma_{1}\right), \\
& \dot{\Sigma}_{2}=D\left(r^{\circ}-\Sigma_{2}\right), \\
& \dot{x}_{j}=\left(\mu_{j}\left(\Sigma_{1}-\sum_{i=1}^{n} \frac{1}{y_{s i}} x_{i}, \Sigma_{2}-\sum_{i=1}^{n} \frac{1}{y_{r i}} x_{i}, x_{j}\right)-D\right) x_{j}, j=1 . . n .
\end{aligned}
$$

Hence, the solutions of the two first equations are simply :

$$
\begin{aligned}
& \Sigma_{1}(t)=s^{\circ}+\left(\Sigma_{1}(0)-s^{\circ}\right) e^{-D t}, \\
& \Sigma_{2}(t)=r^{\circ}+\left(\Sigma_{2}(0)-r^{\circ}\right) e^{-D t} .
\end{aligned}
$$

Finally, we deduce :

$$
\lim _{t \rightarrow+\infty} s(t)=s^{\circ} \text { and } \lim _{t \rightarrow+\infty} r(t)=r^{\circ}
$$

Remark 2.3 In what follows, we suppose that $\mu_{i}\left(s^{\circ}, r^{\circ}, 0\right)>D$ (i.e. $s^{\circ}>\lambda_{s i}$ and $\left.r^{\circ}>\lambda_{r i}\right)$.

\subsection{A persistence result}

Let us additionally assume that, for all $i \in\{1,2, . ., n\}$,

H3. There exists a real number $h \in] 0,1]$ such that

$$
\begin{aligned}
& p_{i}\left(s, x_{i}\right) \leq p_{i}(s, 0) \frac{1}{\left(1+x_{i}\right)^{h}}, \\
& q_{i}\left(r, x_{i}\right) \leq q_{i}(r, 0) \frac{1}{\left(1+x_{i}\right)^{h}} .
\end{aligned}
$$

H4. The functions $s \mapsto p_{i}(s, 0)$ and $r \mapsto q_{i}(r, 0)$ are, respectively, bounded by two real constants $m_{s i}>0$ and $m_{r i}>0$.

Note that, the functions $p_{i}$ and $q_{i}$ being $C^{1}$, there exist two real constants $K_{s i}>0$ and $K_{r i}>0$ such that

$$
p_{i}(s, 0) \leq K_{s i} s \text { and } q_{i}(r, 0) \leq K_{r i} r .
$$


H5. The following inequalities, where $M_{i}:=\min \left(m_{s i}, m_{r i}\right)>D$, are satisfied :

$$
\begin{aligned}
& s^{\circ}>D+\sum_{i=1}^{n} \frac{1}{y_{s i}} K_{s i}\left(\left(\frac{2 M_{i}}{D}\right)^{\frac{1}{h}}-1\right), \\
& r^{\circ}>D+\sum_{i=1}^{n} \frac{1}{y_{r i}} K_{r i}\left(\left(\frac{2 M_{i}}{D}\right)^{\frac{1}{h}}-1\right) .
\end{aligned}
$$

H6. The functions $\mu_{i}$ satisfy $\mu_{i}(D, D, 0)>D$.

Note that the assumption $\mu_{i}\left(s^{\circ}, r^{\circ}, 0\right):=\min \left(p_{i}\left(s^{\circ}, 0\right), q_{i}\left(r^{\circ}, 0\right)\right)>D$ is a consequence of assumptions $\mathrm{H} 5$ and H6.

Remark 2.4 We give an example of a density-dependent growth function which is often used in biology and which satisfies the assumptions $\mathrm{H} 3$ and $\mathrm{H} 4$, namely the Beddington-DeAngelis type growth function :

$$
p(s, x)=\frac{m s}{s+k+a x},
$$

where $m, k$, and $a$ are positive constants. Note that this function satisfies obviously the assumptions $\mathrm{H} 1$ and $\mathrm{H} 2$. One has :

$$
p(s, 0)=\frac{m s}{s+k} .
$$

Assumption $\mathrm{H} 4$ is clearly satisfied by $p(s, 0)$. We show that :

$$
p(s, x)=p(s, 0) \frac{A(s)}{A(s)+x},
$$

with $A(s):=\frac{s+k}{a}$. Now, if $A(s) \leq 1$, it is obvious that :

$$
\frac{A(s)}{A(s)+x} \leq \frac{1}{1+x} .
$$

Hence, we obtain :

$$
p(s, x) \leq p(s, 0) \frac{1}{1+x},
$$

which is the condition $\mathrm{H} 3$ with $h=1$. On the other hand, knowing that $s<s^{\circ}$ we show that if $A(s)>1$ then :

$$
\frac{A(s)}{A(s)+x} \leq \frac{C}{1+x}
$$

where $C:=A\left(s^{\circ}\right)>1$. Hence,

$$
p(s, x) \leq p(s, 0) \frac{C}{1+x} .
$$

Finally, since $C>1$, the two inequalities imply :

$$
p(s, x) \leq p(s, 0) \frac{1}{1+x} .
$$


Now, we can state and prove the main theorem of this section, which gives sufficient conditions of persistence of the model (3).

Theorem 2.5 If assumptions HI to H6 are satisfied, then the model (3) is persistent.

Proof According to assumption $\mathrm{H} 4$, one has $\mu_{i}(s, r, 0) \leq M_{i}$. From the equations of $x_{i}$ in (3) and assumption H3, one deduces that

$$
\dot{x}_{i} \leq\left(\frac{M_{i}}{\left(1+x_{i}\right)^{h}}-D\right) x_{i}
$$

If $\frac{M_{i}}{\left(1+x_{i}\right)^{h}} \leq \frac{D}{2}$ then $\dot{x}_{i} \leq-\frac{D}{2} x_{i}<0$. In other words, if $x_{i} \geq\left(\frac{2 M_{i}}{D}\right)^{\frac{1}{h}}-1$ then $\dot{x}_{i}<0$. Hence,

$$
\exists t_{1}>0 \text { such that } \forall t \geq t_{1} x_{i}(t)<\left(\frac{2 M_{i}}{D}\right)^{\frac{1}{h}}-1
$$

Note that $\left(\frac{2 M_{i}}{D}\right)^{\frac{1}{h}}>1$ since $M_{i}>D$. In the other hand, for all $t \geq t_{1}$, one has

$$
\begin{aligned}
& \dot{s}>\left(s^{\circ}-s\right) D-\sum_{i=1}^{n} \frac{1}{y_{s i}} \mu_{i}\left(s, r, x_{i}\right)\left(\left(\frac{2 M_{i}}{D}\right)^{\frac{1}{h}}-1\right), \\
& \dot{r}>\left(r^{\circ}-r\right) D-\sum_{i=1}^{n} \frac{1}{y_{r i}} \mu_{i}\left(s, r, x_{i}\right)\left(\left(\frac{2 M_{i}}{D}\right)^{\frac{1}{h}}-1\right) .
\end{aligned}
$$

According to assumption $\mathrm{H} 2$, one can see that $\mu_{i}\left(s, r, x_{i}\right) \leq \mu_{i}(s, r, 0)$. Hence,

$$
\begin{aligned}
& \dot{s}>\left(s^{\circ}-s\right) D-\sum_{i=1}^{n} \frac{1}{y_{s i}} \mu_{i}(s, r, 0)\left(\left(\frac{2 M_{i}}{D}\right)^{\frac{1}{h}}-1\right), \\
& \dot{r}>\left(r^{\circ}-r\right) D-\sum_{i=1}^{n} \frac{1}{y_{r i}} \mu_{i}(s, r, 0)\left(\left(\frac{2 M_{i}}{D}\right)^{\frac{1}{h}}-1\right) .
\end{aligned}
$$

Moreover, from $\mathrm{H} 4$, we know that

$$
\begin{aligned}
& \mu_{i}(s, r, 0) \leq p_{i}(s, 0) \leq K_{s i} s, \\
& \mu_{i}(s, r, 0) \leq q_{i}(r, 0) \leq K_{r i} r .
\end{aligned}
$$

Therefore,

$$
\begin{aligned}
& \dot{s}>\left(s^{\circ}-s\right) D-\sum_{i=1}^{n} \frac{1}{y_{s i}} K_{s i}\left(\left(\frac{2 M_{i}}{D}\right)^{\frac{1}{h}}-1\right) s, \\
& \dot{r}>\left(r^{\circ}-r\right) D-\sum_{i=1}^{n} \frac{1}{y_{r i}} K_{r i}\left(\left(\frac{2 M_{i}}{D}\right)^{\frac{1}{h}}-1\right) r .
\end{aligned}
$$


If $s, r \in] 0, D]$, then

$$
\begin{aligned}
& \dot{s}>\left[s^{\circ}-D-\sum_{i=1}^{n} \frac{1}{y_{s i}} K_{s i}\left(\left(\frac{2 M_{i}}{D}\right)^{\frac{1}{h}}-1\right)\right] s, \\
& \dot{r}>\left[r^{\circ}-D-\sum_{i=1}^{n} \frac{1}{y_{r i}} K_{r i}\left(\left(\frac{2 M_{i}}{D}\right)^{\frac{1}{h}}-1\right)\right] r .
\end{aligned}
$$

According to assumption $\mathrm{H} 5$, one concludes that $\dot{s}>0$ and $\dot{r}>0$ for all $s, r \in] 0, D]$. Hence,

$$
\exists t_{2}>t_{1}: \forall t \geq t_{2}, s(t)>D \text { and } \exists t_{2}^{\prime}>t_{1}: \forall t \geq t_{2}^{\prime}, r(t)>D,
$$

then

$$
\forall t \geq \max \left(t_{2}, t_{2}^{\prime}\right), \dot{x}_{i}=\left(\mu_{i}\left(s, r, x_{i}\right)-D\right) x_{i}>\left(\mu_{i}\left(D, D, x_{i}\right)-D\right) x_{i}
$$

Now, by continuity of the function $\mu_{i}$ with respect to $x_{i}$, for all $\varepsilon_{i}>0$, there exists $\delta>0$ such that $\left|\mu_{i}\left(D, D, x_{i}\right)-\mu_{i}(D, D, 0)\right| \leq \varepsilon_{i}$ for all $x_{i} \leq \delta$. Hence, for all $x_{i} \leq \delta$ one has $\mu_{i}\left(D, D, x_{i}\right)-D \geq \mu_{i}(D, D, 0)-\varepsilon_{i}-D$.

Let $A_{i}:=\mu_{i}(D, D, 0)-D$ which is positive according to H6. If we chose $\varepsilon_{i}=\frac{A_{i}}{2}$, then there exists $\delta>0$ such that, for all $x_{i} \leq \delta$,

$$
\mu_{i}\left(D, D, x_{i}\right)-D \geq \frac{A_{i}}{2},
$$

which implies that

$$
\forall x_{i} \leq \delta, \dot{x}_{i}>\frac{A_{i}}{2} x_{i}>0
$$

Finally,

$$
\exists t_{3}>\max \left(t_{2}, t_{2}^{\prime}\right): \forall t \geq t_{3}, x_{i}(t)>\delta
$$

which establishes the persistence of the model (3).

\section{Global asymptotic stability in case of two species}

Consider the model

$$
\begin{aligned}
\dot{s} & =\left(s^{\circ}-s\right) D-\sum_{i=1}^{2} \frac{1}{y_{s i}} \mu_{i}\left(s, r, x_{i}\right) x_{i}, \\
\dot{r} & =\left(r^{\circ}-r\right) D-\sum_{i=1}^{2} \frac{1}{y_{r i}} \mu_{i}\left(s, r, x_{i}\right) x_{i}, \\
\dot{x}_{1} & =\left(\mu_{1}\left(s, r, x_{1}\right)-D\right) x_{1}, \\
\dot{x}_{2} & =\left(\mu_{2}\left(s, r, x_{2}\right)-D\right) x_{2},
\end{aligned}
$$

which is the system (3) with $n=2$ and suppose that assumptions $\mathrm{H} 1$ and $\mathrm{H} 2$ are still satisfied. 


\subsection{Uniform persistence}

In the following Lemma, we omit the subscribe $i$ for convenience.

Lemma 3.1 The equation $\mu\left(s^{\circ}-\frac{1}{y_{s}} x, r^{\circ}-\frac{1}{y_{r}} x, x\right)-D=0$ has a unique solution in ] $0, \min \left(y_{s} s^{\circ}, y_{r} r^{\circ}\right)[$.

Proof Define :

$$
F(x):=\mu\left(s^{\circ}-\frac{1}{y_{s}} x, r^{\circ}-\frac{1}{y_{r}} x, x\right)-D .
$$

One has :

$$
F(0)=\mu\left(s^{\circ}, r^{\circ}, 0\right)-D>0 \text { and } F\left(\min \left(y_{s} s^{\circ}, y_{r} r^{\circ}\right)\right)=-D<0 .
$$

We check now that the function $F$ is decreasing. Recall that both functions $p$ and $q$ are increasing with respect to their first argument and decreasing with respect to the second one. Let $x_{1}<x_{2}$.

$$
\begin{aligned}
p\left(s^{\circ}-\frac{1}{y_{s}} x_{1}, x_{1}\right) & >p\left(s^{\circ}-\frac{1}{y_{s}} x_{2}, x_{1}\right)>p\left(s^{\circ}-\frac{1}{y_{s}} x_{2}, x_{2}\right) \\
& \geqslant \min \left(p\left(s^{\circ}-\frac{1}{y_{s}} x_{2}, x_{2}\right), q\left(r^{\circ}-\frac{1}{y_{r}} x_{2}, x_{2}\right)\right),
\end{aligned}
$$

then

$$
p\left(s^{\circ}-\frac{1}{y_{s}} x_{1}, x_{1}\right)>\mu\left(s^{\circ}-\frac{1}{y_{s}} x_{2}, r^{\circ}-\frac{1}{y_{r}} x_{2}, x_{2}\right) .
$$

Likewise,

$$
\begin{aligned}
q\left(r^{\circ}-\frac{1}{y_{r}} x_{1}, x_{1}\right) & >q\left(r^{\circ}-\frac{1}{y_{r}} x_{2}, x_{1}\right)>q\left(r^{\circ}-\frac{1}{y_{r}} x_{2}, x_{2}\right) \\
& \geqslant \min \left(p\left(s^{\circ}-\frac{1}{y_{s}} x_{2}, x_{2}\right), q\left(r^{\circ}-\frac{1}{y_{r}} x_{2}, x_{2}\right)\right),
\end{aligned}
$$

then

$$
q\left(r^{\circ}-\frac{1}{y_{r}} x_{1}, x_{1}\right)>\mu\left(s^{\circ}-\frac{1}{y_{s}} x_{2}, r^{\circ}-\frac{1}{y_{r}} x_{2}, x_{2}\right) .
$$

These two inequalities imply

$$
\mu\left(s^{\circ}-\frac{1}{y_{s}} x_{1}, r^{\circ}-\frac{1}{y_{r}} x_{1}, x_{1}\right)>\mu\left(s^{\circ}-\frac{1}{y_{s}} x_{2}, r^{\circ}-\frac{1}{y_{r}} x_{2}, x_{2}\right) .
$$

We deduce that $F\left(x_{1}\right)>F\left(x_{2}\right)$. Hence, the equation $F(x)=0$ has a unique solution in ] $0, \min \left(y_{s} s^{\circ}, y_{r} r^{\circ}\right)[$.

We can make the additional following assumption : 
H7. The unique solution $\tilde{x}_{i}$ of the equation $\mu_{i}\left(s^{\circ}-\frac{1}{y_{s i}} x_{i}, r^{\circ}-\frac{1}{y_{r i}} x_{i}, x_{i}\right)-D=0$ in ] $0, \min \left(y_{s i} s^{\circ}, y_{r i} r^{\circ}\right)[$ verifies :

$$
\begin{aligned}
& \mu_{1}\left(s^{\circ}-\frac{\tilde{x}_{2}}{y_{s 2}}, r^{\circ}-\frac{\tilde{x}_{2}}{y_{r 2}}, 0\right)-D>0, \\
& \mu_{2}\left(s^{\circ}-\frac{\tilde{x}_{1}}{y_{s 1}}, r^{\circ}-\frac{\tilde{x}_{1}}{y_{r 1}}, 0\right)-D>0 .
\end{aligned}
$$

Remark 3.2 The assumption $\mu_{i}\left(s^{\circ}, r^{\circ}, 0\right):=\min \left(p_{i}\left(s^{\circ}, 0\right), q_{i}\left(r^{\circ}, 0\right)\right)>D$ is a consequence of the assumption H7 (see Remark 2.3).

Concerning the function $\mu_{i}$ satisfying hypothesis H7, we reconsider here the example of Beddington-DeAngelis growth function and show that the solutions $\tilde{x}_{i}$ can be explicitly determined. This can be done also for the so-called Contois growth function $p(s, x)=$ $m s /(s+k x)$, where $m, k>0$, but, unfortunately, assumption $\mathrm{H} 4$ is not satisfied in this case. For convenience, we will consider the equation :

$$
\mu\left(s^{\circ}-\alpha x, r^{\circ}-\beta x, x\right)-D=0,
$$

where $\alpha=1 / y_{s}$ and $\beta=1 / y_{r}$.

Let us set :

$$
F(x):=\mu\left(s^{\circ}-\alpha x, r^{\circ}-\beta x, x\right)-D,
$$

with

$$
\mu(s, r, x):=\min (p(s, x), q(r, x)) .
$$

Consider then

$$
p(s, x)=\frac{m s}{s+k+a x}, q(r, x)=\frac{m^{\prime} r}{r+k^{\prime}+a^{\prime} x},
$$

where $m, m^{\prime}, k, k^{\prime}, a$ and $a^{\prime}$ are positive constants. If $F(x)=p\left(s^{\circ}-\alpha x, x\right)-D$, then :

$$
\begin{aligned}
F(x)=0 & \Leftrightarrow(\alpha(m-D)+a D) x=(m-D) s^{\circ}-k D \\
& \Leftrightarrow x=\frac{(m-D) s^{\circ}-k D}{\alpha(m-D)+a D}>0 .
\end{aligned}
$$

The positiveness of $x$ is a consequence of the condition of non-washing out (Remark 2.3). The case $F(x)=q\left(r^{\circ}-\beta x, x\right)-D$ is similar.

We can state the following result giving sufficient conditions of permanence of the system (5). The proof will use a corollary 2 of the paper [7] cited in the appendix. This corollary is a consequence of Hofbauer's and Hutson's theorem [9,11].

Theorem 3.3 If assumptions H1, H2 and H7 are satisfied, then the model (5) is permanent.

Proof According to Proposition 2.1, the system (5) is dissipative and the following bounded set

$$
C:=\left\{\left(s, r, x_{1}, x_{2}\right) \in \mathbb{R}_{+}^{4}: s \leq s^{\circ}, r \leq r^{\circ}, x_{i} \leq \min \left(y_{s i} s^{\circ}, y_{r i} r^{\circ}\right)\right\}
$$


is an attracting one, in the sense that all trajectories eventually lie in it. By setting :

$$
\begin{aligned}
& \Sigma_{1}:=s+\sum_{i=1}^{2} \frac{1}{y_{s i}} x_{i}, \\
& \Sigma_{2}:=r+\sum_{i=1}^{2} \frac{1}{y_{r i}} x_{i},
\end{aligned}
$$

the system (5) becomes :

$$
\begin{aligned}
& \dot{\Sigma}_{1}=D\left(s^{\circ}-\Sigma_{1}\right), \\
& \dot{\Sigma}_{2}=D\left(r^{\circ}-\Sigma_{2}\right), \\
& \dot{x}_{1}=\left(\mu_{1}\left(\Sigma_{1}-\sum_{i=1}^{2} \frac{1}{y_{s i}} x_{i}, \Sigma_{2}-\sum_{i=1}^{2} \frac{1}{y_{r i}} x_{i}, x_{1}\right)-D\right) x_{1}, \\
& \dot{x}_{2}=\left(\mu_{2}\left(\Sigma_{1}-\sum_{i=1}^{2} \frac{1}{y_{s i}} x_{i}, \Sigma_{2}-\sum_{i=1}^{2} \frac{1}{y_{r i}} x_{i}, x_{2}\right)-D\right) x_{2} .
\end{aligned}
$$

The solutions of the two first equations are :

$$
\begin{aligned}
& \Sigma_{1}(t)=s^{\circ}+\left(\Sigma_{1}(0)-s^{\circ}\right) e^{-D t}, \\
& \Sigma_{2}(t)=r^{\circ}+\left(\Sigma_{2}(0)-r^{\circ}\right) e^{-D t} .
\end{aligned}
$$

The couple $\left(\Sigma_{1}(t), \Sigma_{2}(t)\right)$ converges exponentially to $\left(s^{\circ}, r^{\circ}\right)$. Hence, the $\omega$-limit set of all positive trajectories of the model (5) lies in the simplex :

$$
\Lambda:=\left\{\left(s, r, x_{1}, x_{2}\right) \in C: s+\sum_{i=1}^{2} \frac{1}{y_{s i}} x_{i}=s^{\circ}, r+\sum_{i=1}^{2} \frac{1}{y_{r i}} x_{i}=r^{\circ}\right\} .
$$

The system (6) reduced on $\Lambda$ is given by :

$$
\begin{aligned}
& \dot{x}_{1}=\left(\mu_{1}\left(s^{\circ}-\sum_{i=1}^{2} \frac{1}{y_{s i}} x_{i}, r^{\circ}-\sum_{i=1}^{2} \frac{1}{y_{r i}} x_{i}, x_{1}\right)-D\right) x_{1}, \\
& \dot{x}_{2}=\left(\mu_{2}\left(s^{\circ}-\sum_{i=1}^{2} \frac{1}{y_{s i}} x_{i}, r^{\circ}-\sum_{i=1}^{2} \frac{1}{y_{r i}} x_{i}, x_{2}\right)-D\right) x_{2},
\end{aligned}
$$

and is defined on :

$$
X:=\left\{\left(x_{1}, x_{2}\right) \in \mathbb{R}_{+}^{2}: \sum_{i=1}^{2} \frac{1}{y_{s i}} x_{i} \leq s^{\circ} \text { and } \sum_{i=1}^{2} \frac{1}{y_{r i}} x_{i} \leq r^{\circ}\right\} .
$$

$X$ is obviously a closed set. Let us show that it is positively invariant. Suppose that $\sum_{i=1}^{2} \frac{1}{y_{s i}} x_{i}(0)=s^{\circ}$ and $\sum_{i=1}^{2} \frac{1}{y_{r i}} x_{i}(0)=r^{\circ}$. One can see that :

$$
\left.\frac{\mathrm{d}}{\mathrm{d} t}\left(\sum_{i=1}^{2} \frac{1}{y_{s i}} x_{i}(t)\right)\right|_{t=0}=-D s^{\circ}<0,\left.\quad \frac{\mathrm{d}}{\mathrm{d} t}\left(\sum_{i=1}^{2} \frac{1}{y_{r i}} x_{i}(t)\right)\right|_{t=0}=-D r^{\circ}<0 .
$$


Hence, for all $t>0$ :

$$
\sum_{i=1}^{2} \frac{1}{y_{s i}} x_{i}(t)<s^{\circ} \text { and } \sum_{i=1}^{2} \frac{1}{y_{r i}} x_{i}(t)<r^{\circ} .
$$

On the other hand, one has :

$$
x_{i}(t)=x_{i}(0) \exp \left[\int_{0}^{t}\left(\mu_{i}\left(s^{\circ}-\sum_{i=1}^{2} \frac{1}{y_{s i}} x_{i}(\tau), r^{\circ}-\sum_{i=1}^{2} \frac{1}{y_{r i}} x_{i}(\tau), x_{i}(\tau)\right)-D\right) \mathrm{d} \tau\right] .
$$

Hence, $x_{i}(t) \geq 0$ when $x_{i}(0) \geq 0$.

Now, let set $S:=\partial R_{+}^{2} \cap X=\left[0, \min \left(y_{s 1} s^{\circ}, y_{r 1} r^{\circ}\right)\right] \cup\left[0, \min \left(y_{s 2} s^{\circ}, y_{r 2} r^{\circ}\right)\right]$. The set $S$ is obviously compact and positively invariant. Define the function $P: X \rightarrow \mathbb{R}_{+}$by $P\left(x_{1}, x_{2}\right):=x_{1} x_{2}$, which clearly vanishes on $S$. We compute the derivative of $P$ at $\left(x_{1}, x_{2}\right) \in$ $X \backslash S$ in the direction of the field $F$ defined by the reduced system (7).

$$
\begin{aligned}
\dot{P}\left(x_{1}, x_{2}\right) & :=\left\langle\operatorname{grad} P\left(x_{1}, x_{2}\right), F\left(x_{1}, x_{2}\right)\right\rangle=\sum_{j=1}^{2} \frac{\partial P\left(x_{1}, x_{2}\right)}{\partial x_{j}} F_{j}\left(x_{1}, x_{2}\right) \\
& =x_{1} x_{2}\left[\sum_{i=1}^{2} \mu_{i}\left(s^{\circ}-\sum_{i=1}^{2} \frac{1}{y_{s i}} x_{i}, r^{\circ}-\sum_{i=1}^{2} \frac{1}{y_{r i}} x_{i}, x_{i}\right)-2 D\right] .
\end{aligned}
$$

Let $\psi: X \longrightarrow \mathbb{R}$ defined by :

$$
\psi\left(x_{1}, x_{2}\right):=\sum_{i=1}^{2} \mu_{i}\left(s^{\circ}-\sum_{i=1}^{2} \frac{1}{y_{s i}} x_{i}, r^{\circ}-\sum_{i=1}^{2} \frac{1}{y_{r i}} x_{i}, x_{i}\right)-2 D .
$$

One has :

$$
\dot{P}\left(x_{1}, x_{2}\right)=P\left(x_{1}, x_{2}\right) \psi\left(x_{1}, x_{2}\right) \quad \forall\left(x_{1}, x_{2}\right) \in X \backslash S .
$$

The function $\psi$ is bounded below by $-2 D$. The condition $(i)$ of Corollary A.1 of the appendix is then verified. For the condition $(i i)$, it is sufficient to check that $\psi\left(E_{k}\right)>0$ where $E_{k}$ is an equilibrium point in $S$. Indeed, the unidimensional set $S$ is positively invariant and its $\omega$-limit set, denoted by $\Omega(S)$, is formed by the equilibrium points $E_{k}$ in $S$. Let us determine these equilibrium points.

If $\left(x_{1}(0), x_{2}(0)\right) \in S$ such that $x_{1}(0)>0$ and $x_{2}(0)=0$, then $x_{2}(t) \equiv 0$ and the system (7) is written :

$$
\dot{x}_{1}=\left(g_{1}\left(s^{\circ}-\frac{1}{y_{s 1}} x_{1}, r^{\circ}-\frac{1}{y_{r 1}} x_{1}, x_{1}\right)-D\right) x_{1} .
$$

The nullclines are $g_{1}\left(s^{\circ}-\frac{1}{y_{s}} x_{1}, r^{\circ}-\frac{1}{y_{r}} x_{1}, x_{1}\right)-D=0$ or $x_{1}=0$. If $x_{1}=0$, then $E_{0}(0,0)$ is an equilibrium point in $S$. If $x_{1} \neq 0$, then according to assumption $\mathrm{H} 7$, the first equation has a unique solution $\tilde{x}_{1}$ in $] 0, \min \left(y_{s 1} s^{\circ}, y_{r 1} r^{\circ}\right)$ [. Hence, $E_{1}\left(\tilde{x}_{1}, 0\right)$ is an equilibrium point in $S$. Analogously, if $\left(x_{1}(0), x_{2}(0)\right) \in S$ such that $x_{1}(0)=0$ and $x_{2}(0)>0$, then there exists another equilibrium point $E_{2}\left(0, \tilde{x}_{2}\right)$ for $\left.\tilde{x}_{2} \in\right] 0, \min \left(y_{s 2} s^{\circ}, y_{r 2} r^{\circ}\right)$. Hence, $\Omega(S)=\left\{E_{0}, E_{1}, E_{2}\right\}$. From assumption $\mathrm{H} 7$, one has : 


$$
\begin{aligned}
& \psi\left(E_{0}\right)=\sum_{i=1}^{2} \mu_{i}\left(s^{\circ}, r^{\circ}, 0\right)-2 D>0, \\
& \psi\left(E_{1}\right)=\mu_{2}\left(s^{\circ}-\frac{\tilde{x}_{1}}{y_{s 1}}, r^{\circ}-\frac{\tilde{x}_{1}}{y_{r 1}}, 0\right)-D>0, \\
& \psi\left(E_{2}\right)=\mu_{1}\left(s^{\circ}-\frac{\tilde{x}_{2}}{y_{s 2}}, r^{\circ}-\frac{\tilde{x}_{2}}{y_{r 2}}, 0\right)-D>0 .
\end{aligned}
$$

The condition (ii) of Corollary A.1 is then satisfied which implies that $S$ is a uniform repeller and that the model (7) is uniformly persistent. Finally, since $\lim _{t \rightarrow+\infty} \Sigma_{1}(t)=s^{\circ}$ and $\lim _{t \rightarrow+\infty} \Sigma_{2}(t)=r^{\circ}$, the whole system (5) is uniformly persistent.

\subsection{Global asymptotic stability}

Uniform persistence and properties of the planar systems lead to the following result of existence of a G.A.S. equilibrium point.

Theorem 3.4 Under assumptions H1, H2 and H7, the system (5) has a positive G.A.S. equilibrium point.

Proof First, we determine the trivial equilibrium points of the reduced system (7) and their nature. The Jacobian matrix at an arbitrary point $E\left(x_{1}, x_{2}\right)$ is given by :

$$
J(E)=\left[\begin{array}{cc}
\mu_{1}-D+x_{1} a_{1} & x_{1} b_{12} \\
x_{2} b_{21} & \mu_{2}-D+x_{2} a_{2}
\end{array}\right],
$$

with the following notations :

$$
\begin{aligned}
\mu_{j} & :=\mu_{j}\left(s^{\circ}-\sum_{i=1}^{2} \frac{1}{y_{s i}} x_{i}, r^{\circ}-\sum_{i=1}^{2} \frac{1}{y_{r i}} x_{i}, x_{j}\right), \\
a_{j} & :=-\frac{1}{y_{s j}} \frac{\partial p_{j}}{\partial s}\left(s^{\circ}-\sum_{i=1}^{2} \frac{1}{y_{s i}} x_{i}, x_{j}\right)+\frac{\partial p_{j}}{\partial x_{j}}\left(s^{\circ}-\sum_{i=1}^{2} \frac{1}{y_{s i}} x_{i}, x_{j}\right) \text { or } \\
a_{j} & :=-\frac{1}{y_{r j}} \frac{\partial q_{j}}{\partial r}\left(r^{\circ}-\sum_{i=1}^{2} \frac{1}{y_{s i}} x_{i}, x_{j}\right)+\frac{\partial q_{j}}{\partial x_{j}}\left(r^{\circ}-\sum_{i=1}^{2} \frac{1}{y_{s i}} x_{i}, x_{j}\right), \\
b_{j k} & :=-\frac{1}{y_{s k}} \frac{\partial p_{j}}{\partial s}\left(s^{\circ}-\sum_{i=1}^{2} \frac{1}{y_{s i}} x_{i}, x_{j}\right) \text { or } \\
b_{j k} & :=-\frac{1}{y_{r k}} \frac{\partial q_{j}}{\partial r}\left(r^{\circ}-\sum_{i=1}^{2} \frac{1}{y_{s i}} x_{i}, x_{j}\right) \text { with } k \in\{1,2\}, k \neq j .
\end{aligned}
$$

The nullclines equations are given by :

$$
\begin{aligned}
& \left(\mu_{1}\left(s^{\circ}-\sum_{i=1}^{2} \frac{1}{y_{s i}} x_{i}, r^{\circ}-\sum_{i=1}^{2} \frac{1}{y_{r i}} x_{i}, x_{1}\right)-D\right) x_{1}=0, \\
& \left(\mu_{2}\left(s^{\circ}-\sum_{i=1}^{2} \frac{1}{y_{s i}} x_{i}, r^{\circ}-\sum_{i=1}^{2} \frac{1}{y_{r i}} x_{i}, x_{2}\right)-D\right) x_{2}=0 .
\end{aligned}
$$


$\mathbf{1}^{\text {st }}$ case : $x_{1}=x_{2}=0$. The Jacobian matrix at the equilibrium point $E_{0}(0,0)$ is simply :

$$
J\left(E_{0}\right)=\left[\begin{array}{cc}
\mu_{1}\left(s^{\circ}, r^{\circ}, 0\right)-D & 0 \\
0 & \mu_{2}\left(s^{\circ}, r^{\circ}, 0\right)-D
\end{array}\right],
$$

the eigenvalues of which are $v_{i}^{0}:=\mu_{i}\left(s^{\circ}, r^{\circ}, 0\right)-D, i \in\{1,2\}$. The origin $E_{0}$ unstable, since $\mu_{i}\left(s^{\circ}, r^{\circ}\right)>D$.

$2^{\text {nd }}$ case : $x_{1} \neq 0, x_{2}=0$. According to assumption $\mathrm{H} 7$, the equation :

$$
\mu_{1}\left(s^{\circ}-\frac{1}{y_{s 1}} x_{1}, r^{\circ}-\frac{1}{y_{r 1}} x_{1}, x_{1}\right)-D=0,
$$

has a unique solution $\tilde{x}_{1}$ in $] 0, \min \left(y_{s} s^{\circ}, y_{r 1} r^{\circ}\right)[$. The Jacobian matrix evaluated at the equilibrium point $E_{1}\left(\tilde{x}_{1}, 0\right)$ is given by :

$$
J\left(E_{1}\right)=\left[\begin{array}{cc}
\tilde{x}_{1} \tilde{a}_{1} & \tilde{x}_{1} \tilde{b}_{12} \\
0 & \tilde{\mu}_{2}-D
\end{array}\right],
$$

where

$$
\begin{aligned}
\tilde{\mu}_{2} & :=\mu_{2}\left(s^{\circ}-\frac{1}{y_{s 1}} \tilde{x}_{1}, r^{\circ}-\frac{1}{y_{r 1}} \tilde{x}_{1}, 0\right), \\
\tilde{a}_{1} & :=-\frac{1}{y_{s 1}} \frac{\partial p_{1}}{\partial s}\left(s^{\circ}-\frac{1}{y_{s 1}} \tilde{x}_{1}, \tilde{x}_{1}\right)+\frac{\partial p_{1}}{\partial x_{1}}\left(s^{\circ}-\frac{1}{y_{s 1}} \tilde{x}_{1}, \tilde{x}_{1}\right) \text { or } \\
\tilde{a}_{1} & :=-\frac{1}{y_{r 1}} \frac{\partial q_{1}}{\partial r}\left(r^{\circ}-\frac{1}{y_{r 1}} \tilde{x}_{1}, \tilde{x}_{1}\right)+\frac{\partial q_{1}}{\partial x_{1}}\left(r^{\circ}-\frac{1}{y_{r 1}} \tilde{x}_{1}, \tilde{x}_{1}\right), \\
\tilde{b}_{12} & :=-\frac{1}{y_{s 2}} \frac{\partial p_{1}}{\partial s}\left(s^{\circ}-\frac{1}{y_{s 1}} \tilde{x}_{1}, \tilde{x}_{1}\right) \text { or } \\
\tilde{b}_{12} & :=-\frac{1}{y_{r 2}} \frac{\partial q_{1}}{\partial r}\left(r^{\circ}-\frac{1}{y_{r 1}} \tilde{x}_{1}, \tilde{x}_{1}\right) .
\end{aligned}
$$

The eigenvalues of $J\left(E_{1}\right)$ are $v_{1}^{1}:=\tilde{x}_{1} \tilde{a}_{1}$ and $v_{2}^{1}:=\tilde{\mu}_{2}-D$. According to assumption $\mathrm{H} 2$, the value $v_{1}^{1}$ is negative while, according to assumption $\mathrm{H} 7$, the value $v_{2}^{1}$ is positive. The equilibrium point $E_{1}$ is unstable (saddle point).

$3^{r d}$ case : $x_{1}=0, x_{2} \neq 0$. This case is analogous to the second one. The obtained equilibrium point, also a saddle point, is $E_{2}\left(0, \tilde{x}_{2}\right)$ where $\tilde{x}_{2}$ is the unique solution of :

$$
\mu_{2}\left(s^{\circ}-\frac{1}{y_{s 2}} x_{2}, r^{\circ}-\frac{1}{y_{r 2}} x_{2}, x_{2}\right)-D=0,
$$

in $] 0, \min \left(y_{s 2} s^{\circ}, y_{r 2} r^{\circ}\right)[$.

Now, let us show that the model (7) has no limit cycle with the use of Dulac-Bendixson Criterion [18]. Let $A$ be the simply connected attracting domain of the system (7):

$$
A:=\left\{\left(x_{1}, x_{2}\right) \in \mathbb{R}_{+}^{2}: \varepsilon \leq x_{i} \leq \min \left(y_{s i} s^{\circ}, y_{r i} r^{\circ}\right)\right\},
$$

where $\varepsilon$ is a positive real number such that $\liminf _{t \rightarrow+\infty} x_{i}(t)>\varepsilon$. Such a uniform bound exists according the Theorem 3.3. Let $B: A \longrightarrow \mathbb{R}$ the $C^{1}$ function defined by $B\left(x_{1}, x_{2}\right):=$ $1 / x_{1} x_{2}$ and denote by $F:=\left(F_{1}, F_{2}\right)$ the vector field of (7): 


$$
\begin{aligned}
& F_{1}\left(x_{1}, x_{2}\right):=\left(\mu_{1}\left(s^{\circ}-\sum_{i=1}^{2} \frac{1}{y_{s i}} x_{i}, r^{\circ}-\sum_{i=1}^{2} \frac{1}{y_{r i}} x_{i}, x_{1}\right)-D\right) x_{1}, \\
& F_{2}\left(x_{1}, x_{2}\right):=\left(\mu_{2}\left(s^{\circ}-\sum_{i=1}^{2} \frac{1}{y_{s i}} x_{i}, r^{\circ}-\sum_{i=1}^{2} \frac{1}{y_{r i}} x_{i}, x_{2}\right)-D\right) x_{2} .
\end{aligned}
$$

The divergence of the field $B . F$ is then given by :

$$
\operatorname{div}\left((B . F)\left(x_{1}, x_{2}\right)\right):=\frac{\partial}{\partial x_{1}}\left(B\left(x_{1}, x_{2}\right) F_{1}\left(x_{1}, x_{2}\right)\right)+\frac{\partial}{\partial x_{2}}\left(B\left(x_{1}, x_{2}\right) F_{2}\left(x_{1}, x_{2}\right)\right) .
$$

Simple calculations lead to

$$
\frac{\partial}{\partial x_{1}}\left(B\left(x_{1}, x_{2}\right) F_{1}\left(x_{1}, x_{2}\right)\right)=\frac{1}{x_{2}} a_{1} \text { and } \frac{\partial}{\partial x_{2}}\left(B\left(x_{1}, x_{2}\right) F_{2}\left(x_{1}, x_{2}\right)\right)=\frac{1}{x_{1}} a_{2},
$$

where

$$
\begin{aligned}
& a_{j}:=-\frac{1}{y_{s j}} \frac{\partial p_{j}}{\partial s}\left(s^{\circ}-\sum_{i=1}^{2} \frac{1}{y_{s i}} x_{i}, x_{j}\right)+\frac{\partial p_{j}}{\partial x_{j}}\left(s^{\circ}-\sum_{i=1}^{2} \frac{1}{y_{s i}} x_{i}, x_{j}\right) \text { or } \\
& a_{j}:=-\frac{1}{y_{r j}} \frac{\partial q_{j}}{\partial r}\left(r^{\circ}-\sum_{i=1}^{2} \frac{1}{y_{s i}} x_{i}, x_{j}\right)+\frac{\partial q_{j}}{\partial x_{j}}\left(r^{\circ}-\sum_{i=1}^{2} \frac{1}{y_{s i}} x_{i}, x_{j}\right) .
\end{aligned}
$$

Finally,

$$
\operatorname{div}\left((B . F)\left(x_{1}, x_{2}\right)\right)=\frac{1}{x_{2}} a_{1}+\frac{1}{x_{1}} a_{2} .
$$

According to assumption $\mathrm{H} 2$, the quantity $\operatorname{div}\left((B . F)\left(x_{1}, x_{2}\right)\right)$ is negative for all $\left(x_{1}, x_{2}\right)$ in $A$. Hence, according to Dulac-Bendixson Criterion and Poincaré-Bendixson theorem, the model (7) has no limit cycle and admits at least a positive equilibrium point in $A$. Let $E\left(x_{1}^{*}, x_{2}^{*}\right)$ be such a point and let us evaluate the Jacobian matrix at it :

$$
J(E)=\left[\begin{array}{cc}
x_{1}^{*} a_{1}^{*} & x_{1}^{*} b_{12}^{*} \\
x_{2}^{*} b_{21}^{*} & x_{2}^{*} a_{2}^{*}
\end{array}\right]
$$

where :

$$
\begin{aligned}
& a_{j}^{*}:=-\frac{1}{y_{s j}} \frac{\partial p_{j}}{\partial s}\left(s^{\circ}-\sum_{i=1}^{2} \frac{1}{y_{s i}} x_{i}^{*}, x_{j}^{*}\right)+\frac{\partial p_{j}}{\partial x_{j}}\left(s^{\circ}-\sum_{i=1}^{2} \frac{1}{y_{s i}} x_{i}^{*}, x_{j}^{*}\right) \text { or } \\
& a_{j}^{*}:=-\frac{1}{y_{r j}} \frac{\partial q_{j}}{\partial r}\left(r^{\circ}-\sum_{i=1}^{2} \frac{1}{y_{s i}} x_{i}^{*}, x_{j}^{*}\right)+\frac{\partial q_{j}}{\partial x_{j}}\left(r^{\circ}-\sum_{i=1}^{2} \frac{1}{y_{s i}} x_{i}^{*}, x_{j}^{*}\right), \\
& b_{j k}^{*}:=-\frac{1}{y_{s k}} \frac{\partial p_{j}}{\partial s}\left(s^{\circ}-\sum_{i=1}^{2} \frac{1}{y_{s i}} x_{i}^{*}, x_{j}^{*}\right) \text { or } \\
& b_{j k}^{*}:=-\frac{1}{y_{r k}} \frac{\partial q_{j}}{\partial r}\left(r^{\circ}-\sum_{i=1}^{2} \frac{1}{y_{s i}} x_{i}^{*}, x_{j}^{*}\right) \text { with } k \in\{1,2\}, k \neq j .
\end{aligned}
$$

The trace $\operatorname{Tr}(J(E))=x_{1}^{*} a_{1}^{*}+x_{2}^{*} a_{2}^{*}$ of $J(E)$ is clearly negative. The determinant is given by $\operatorname{det}(J(E))=x_{1}^{*} x_{2}^{*}\left(a_{1}^{*} a_{2}^{*}-b_{12}^{*} b_{21}^{*}\right)$. If, for example, at the equilibrium point, $\mu_{j}=p_{j}($ the case $\mu_{j}=q_{j}$ is similar), one has : 


$$
\operatorname{det}(J(E))=\frac{\partial p_{1}}{\partial x_{1}} \frac{\partial p_{2}}{\partial x_{2}}-\frac{1}{y_{s} 1} \frac{\partial p_{1}}{\partial s} \frac{\partial p_{2}}{\partial x_{2}}-\frac{1}{y_{s} 2} \frac{\partial p_{2}}{\partial s} \frac{\partial p_{1}}{\partial x_{1}}>0
$$

The equilibrium point $E$ is then locally asymptotically stable. Therefore, knowing that there is no limit cycle, and that the equilibrium $E$ is an arbitrary one, which is locally asymptotically stable, one can conclude that $E$ is unique and G.A.S..

Return back to the whole system (6). In the proof of Theorem 3.3, we showed that the equilibrium point $E_{s r}\left(s^{\circ}, r^{\circ}\right)$ is G.A.S. for the first two equations of the model (6). Moreover, the solutions of (6) are positively bounded. Then, one can use the Separation Principle Lemma [21] to conclude that the positive equilibrium point $E^{*}\left(s^{\circ}, r^{\circ}, x_{1}^{*}, x_{2}^{*}\right)$ of the model (6), then also the positive equilibrium point $\left(s^{\circ}-\sum_{i=1}^{2} \frac{1}{y_{s i}} x_{i}^{*}, r^{\circ}-\sum_{i=1}^{2} \frac{1}{y_{r i}} x_{i}^{*}, x_{1}^{*}, x_{2}^{*}\right)$ of the initial model (5), is G.A.S.

\section{Uniform persistence of three species of microorganisms}

Reconsider the model (3) with $n=3$.

$$
\begin{gathered}
\dot{s}=\left(s^{\circ}-s\right) D-\sum_{i=1}^{3} \frac{1}{y_{s i}} \mu_{i}\left(s, r, x_{i}\right) x_{i}, \\
\dot{r}=\left(r^{\circ}-r\right) D-\sum_{i=1}^{3} \frac{1}{y_{r i}} \mu_{i}\left(s, r, x_{i}\right) x_{i}, \\
\dot{x}_{i}=\left(\mu_{i}\left(s, r, x_{i}\right)-D\right) x_{i}, \quad i \in\{1,2,3\} .
\end{gathered}
$$

We show in this section that we are able to say more than for the general case even if we do not prove the existence of a G.A.S. point. We assume that hypotheses H1 to H7 for all $i \in\{1,2,3\}$ are fulfilled, where $\mathrm{H} 7$ should be understood as follows : the unique solution $\tilde{x}_{i}$ of the equation $\mu_{i}\left(s^{\circ}-\frac{1}{y_{s i}} x_{i}, r^{\circ}-\frac{1}{y_{r i}} x_{i}, x_{i}\right)-D=0$ in $] 0, \min \left(y_{s i} s^{\circ}, y_{r i} r^{\circ}\right)[$ satisfies :

$$
\mu_{i}\left(s^{\circ}-\frac{\tilde{x}_{j}}{y_{s j}}, r^{\circ}-\frac{\tilde{x}_{j}}{y_{r j}}, 0\right)-D>0, i, j \in\{1,2,3\}, i \neq j .
$$

We can state and show that :

Theorem 4.1 Under assumptions H1-H7, the model (8) is permanent.

Proof 1. Reduction : According to Proposition 2.1, the system (3) is dissipative and the following set in an attracting one :

$$
C:=\left\{\left(s, r, x_{1}, x_{2}, x_{3}\right) \in \mathbb{R}_{+}^{5}: s \leq s^{\circ}, r \leq r^{\circ}, x_{i} \leq \min \left(y_{s i} s^{\circ}, y_{r i} r^{\circ}\right)\right\}
$$

If we set $\Sigma_{1}:=s+\sum_{i=1}^{3} \frac{1}{y_{s i}} x_{i}$ and $\Sigma_{2}:=r+\sum_{i=1}^{3} \frac{1}{y_{r i}} x_{i}$, the system (8) becomes : 


$$
\begin{aligned}
& \dot{\Sigma}_{1}=D\left(s^{\circ}-\Sigma_{1}\right), \\
& \dot{\Sigma}_{2}=D\left(r^{\circ}-\Sigma_{2}\right), \\
& \dot{x}_{1}=\left(\mu_{1}\left(\Sigma_{1}-\sum_{i=1}^{3} \frac{1}{y_{s i}} x_{i}, \Sigma_{2}-\sum_{i=1}^{3} \frac{1}{y_{r i}} x_{i}, x_{1}\right)-D\right) x_{1}, \\
& \dot{x}_{2}=\left(\mu_{2}\left(\Sigma_{1}-\sum_{i=1}^{3} \frac{1}{y_{s i}} x_{i}, \Sigma_{2}-\sum_{i=1}^{3} \frac{1}{y_{r i}} x_{i}, x_{2}\right)-D\right) x_{2}, \\
& \dot{x}_{3}=\left(\mu_{3}\left(\Sigma_{1}-\sum_{i=1}^{3} \frac{1}{y_{s i}} x_{i}, \Sigma_{2}-\sum_{i=1}^{3} \frac{1}{y_{r i}} x_{i}, x_{3}\right)-D\right) x_{3},
\end{aligned}
$$

the $\omega$-limit set of which lies in the simplex :

$$
\Lambda:=\left\{\left(s, r, x_{1}, x_{2}, x_{3}\right) \in C: s+\sum_{i=1}^{3} \frac{1}{y_{s i}} x_{i}=s^{\circ}, r+\sum_{i=1}^{3} \frac{1}{y_{r i}} x_{i}=r^{\circ}\right\} .
$$

On $\Lambda$ the system (9) is reduced to :

$$
\begin{aligned}
& \dot{x}_{1}=\left(\mu_{1}\left(s^{\circ}-\sum_{i=1}^{3} \frac{1}{y_{s i}} x_{i}, r^{\circ}-\sum_{i=1}^{3} \frac{1}{y_{r i}} x_{i}, x_{1}\right)-D\right) x_{1}, \\
& \dot{x}_{2}=\left(\mu_{2}\left(s^{\circ}-\sum_{i=1}^{3} \frac{1}{y_{s i}} x_{i}, r^{\circ}-\sum_{i=1}^{3} \frac{1}{y_{r i}} x_{i}, x_{2}\right)-D\right) x_{2}, \\
& \dot{x}_{3}=\left(\mu_{3}\left(s^{\circ}-\sum_{i=1}^{3} \frac{1}{y_{s i}} x_{i}, r^{\circ}-\sum_{i=1}^{3} \frac{1}{y_{r i}} x_{i}, x_{3}\right)-D\right) x_{3} .
\end{aligned}
$$

The system (10) is defined on :

$$
X:=\left\{\left(x_{1}, x_{2}, x_{3}\right) \in \mathbb{R}_{+}^{3}: \sum_{i=1}^{3} \frac{1}{y_{s i}} x_{i} \leq s^{\circ} \text { and } \sum_{i=1}^{3} \frac{1}{y_{r i}} x_{i} \leq r^{\circ}\right\} .
$$

2. Boundary equilibria: In order to use Thieme-Zhao's theorem, we set $X_{1}:=$ int $X$ and $X_{2}:=\partial X$. Hence $X=X_{1} \cup X_{2}, X_{1} \cap X_{2}=\emptyset$. The set $X_{2}$ is a closed subset of $X$. Moreover, $X_{1}$ is positively invariant for (10) since, for all positive initial condition, one has :

$x_{i}(t)=x_{i}(0) \exp \left[\int_{0}^{t}\left(\mu_{i}\left(s^{\circ}-\sum_{i=1}^{3} \frac{1}{y_{s i}} x_{i}(\tau), r^{\circ}-\sum_{i=1}^{3} \frac{1}{y_{r i}} x_{i}(\tau), x_{i}(\tau)\right)-D\right) \mathrm{d} \tau\right]>0$.

Now, let us determine the set $M$ of the equilibria of the system (10) in $X_{2}$. First, we claim that any solution of this system starting in $X_{1}$ converges to a point of $M$. Indeed, in the proof of Theorem 2.5, we have already showed that, under assumptions H1-H6, there exists a positive real number $\delta$ and a positive instant $t_{0}$ such that, for all $t \geq t_{0}$ and all $i \in\{1,2, \ldots, n\}$, one has $x_{i}(t)>\delta$. Hence, $\liminf _{t \rightarrow+\infty} x_{i}(t)>0$ for all component $x_{i}(t)$ of a positive solution of the system (10). Now, the jacobian matrix of (10) at an arbitrary point $E\left(x_{1}, x_{2}, x_{3}\right)$ is given by : 


$$
J(E)=\left[\begin{array}{ccc}
\mu_{1}-D+x_{1} a_{1} & x_{1} b_{12} & x_{1} b_{13} \\
x_{2} b_{21} & \mu_{2}-D+x_{2} a_{2} & x_{2} b_{23} \\
x_{3} b_{31} & x_{3} b_{32} & \mu_{3}-D+x_{3} a_{3}
\end{array}\right]
$$

where :

$$
\begin{aligned}
\mu_{j} & :=\mu_{j}\left(s^{\circ}-\sum_{i=1}^{3} \frac{1}{y_{s i}} x_{i}, r^{\circ}-\sum_{i=1}^{3} \frac{1}{y_{r i}} x_{i}, x_{j}\right), \\
a_{j} & :=-\frac{1}{y_{s j}} \frac{\partial p_{j}}{\partial s}\left(s^{\circ}-\sum_{i=1}^{3} \frac{1}{y_{s i}} x_{i}, x_{j}\right)+\frac{\partial p_{j}}{\partial x_{j}}\left(s^{\circ}-\sum_{i=1}^{3} \frac{1}{y_{s i}} x_{i}, x_{j}\right) \text { or } \\
a_{j} & :=-\frac{1}{y_{r j}} \frac{\partial q_{j}}{\partial r}\left(r^{\circ}-\sum_{i=1}^{3} \frac{1}{y_{s i}} x_{i}, x_{j}\right)+\frac{\partial q_{j}}{\partial x_{j}}\left(r^{\circ}-\sum_{i=1}^{3} \frac{1}{y_{s i}} x_{i}, x_{j}\right), \\
b_{j k} & :=-\frac{1}{y_{s k}} \frac{\partial p_{j}}{\partial s}\left(s^{\circ}-\sum_{i=1}^{3} \frac{1}{y_{s i}} x_{i}, x_{j}\right) \text { or } \\
b_{j k} & :=-\frac{1}{y_{r k}} \frac{\partial q_{j}}{\partial r}\left(r^{\circ}-\sum_{i=1}^{3} \frac{1}{y_{s i}} x_{i}, x_{j}\right) \text { with } k \in\{1,2,3\}, k \neq j .
\end{aligned}
$$

The nullclines being :

$$
\begin{aligned}
& \left(\mu_{1}\left(s^{\circ}-\sum_{i=1}^{3} \frac{1}{y_{s i}} x_{i}, r^{\circ}-\sum_{i=1}^{3} \frac{1}{y_{r i}} x_{i}, x_{1}\right)-D\right) x_{1}=0, \\
& \left(\mu_{2}\left(s^{\circ}-\sum_{i=1}^{3} \frac{1}{y_{s i}} x_{i}, r^{\circ}-\sum_{i=1}^{3} \frac{1}{y_{r i}} x_{i}, x_{2}\right)-D\right) x_{2}=0, \\
& \left(\mu_{3}\left(s^{\circ}-\sum_{i=1}^{3} \frac{1}{y_{s i}} x_{i}, r^{\circ}-\sum_{i=1}^{3} \frac{1}{y_{r i}} x_{i}, x_{3}\right)-D\right) x_{3}=0,
\end{aligned}
$$

we distinguish the following cases :

If $x_{1}=x_{2}=x_{3}=0$, the origin $E_{0}(0,0,0) \in X_{2}$ is an equilibrium point at which the jacobian matrix is :

$$
J\left(E_{0}\right)=\left[\begin{array}{ccc}
\mu_{1}\left(s^{\circ}, r^{\circ}, 0\right)-D & 0 & 0 \\
0 & \mu_{2}\left(s^{\circ}, r^{\circ}, 0\right)-D & 0 \\
0 & 0 & \mu_{3}\left(s^{\circ}, r^{\circ}, 0\right)-D
\end{array}\right] .
$$

Since the eigenvalues are all positive, $E_{0}$ is unstable.

If $x_{1} \neq 0, x_{2}=x_{3}=0$, according to assumption $\mathrm{H} 7$, the nullcline

$$
\mu_{1}\left(s^{\circ}-\frac{1}{y_{s 1}} x_{1}, r^{\circ}-\frac{1}{y_{r 1}} x_{1}, x_{1}\right)-D=0,
$$

has a unique solution $\tilde{x}_{1}$ in $] 0, \min \left(y_{s 1} s^{\circ}, y_{r 1} r^{\circ}\right)[$. The jacobian matrix of the system (10) at the boundary equilibrium point $E_{1}\left(\tilde{x}_{1}, 0,0\right) \in X_{2}$ is :

$$
J\left(E_{1}\right)=\left[\begin{array}{ccc}
\tilde{x}_{1} \tilde{a}_{1} & \tilde{x}_{1} \tilde{b}_{12} & \tilde{x}_{1} \tilde{b}_{13} \\
0 & \tilde{\mu}_{2}-D & 0 \\
0 & 0 & \tilde{\mu}_{3}-D
\end{array}\right]
$$


where

$$
\begin{aligned}
\tilde{\mu}_{j} & :=\mu_{j}\left(s^{\circ}-\frac{1}{y_{s 1}} \tilde{x}_{1}, r^{\circ}-\frac{1}{y_{r 1}} \tilde{x}_{1}, 0\right), j \in\{2,3\}, \\
\tilde{a}_{1} & :=-\frac{1}{y_{s 1}} \frac{\partial p_{1}}{\partial s}\left(s^{\circ}-\frac{1}{y_{s 1}} \tilde{x}_{1}, \tilde{x}_{1}\right)+\frac{\partial p_{1}}{\partial x_{1}}\left(s^{\circ}-\frac{1}{y_{s 1}} \tilde{x}_{1}, \tilde{x}_{1}\right) \text { or } \\
\tilde{a}_{1} & :=-\frac{1}{y_{r 1}} \frac{\partial q_{1}}{\partial r}\left(r^{\circ}-\frac{1}{y_{r 1}} \tilde{x}_{1}, \tilde{x}_{1}\right)+\frac{\partial q_{1}}{\partial x_{1}}\left(r^{\circ}-\frac{1}{y_{r 1}} \tilde{x}_{1}, \tilde{x}_{1}\right), \\
\tilde{b}_{1 k} & :=-\frac{1}{y_{s k}} \frac{\partial p_{1}}{\partial s}\left(s^{\circ}-\frac{1}{y_{s 1}} \tilde{x}_{1}, \tilde{x}_{1}\right) \text { or } \\
\tilde{b}_{1 k} & :=-\frac{1}{y_{r k}} \frac{\partial q_{1}}{\partial r}\left(r^{\circ}-\frac{1}{y_{r 1}} \tilde{x}_{1}, \tilde{x}_{1}\right), k \in\{2,3\} .
\end{aligned}
$$

The eigenvalues of $J\left(E_{1}\right)$ are $v_{1}^{1}:=\tilde{x}_{1} \tilde{a}_{1}, v_{2}^{1}:=\tilde{\mu}_{2}-D$ and $v_{3}^{1}:=\tilde{\mu}_{3}-D$. The first one is negative according to $\mathrm{H} 2$, the others are positives according to $\mathrm{H} 7$. The equilibrium $E_{1}$ is then a saddle point. The stable separatrix lies on the $x_{1}$-axis. The other two cases where either $x_{2} \neq 0$ or $x_{3} \neq 0$ is analogous and determines two other saddle points $E_{2}\left(0, \tilde{x}_{2}, 0\right)$ and $E_{3}\left(0,0, \tilde{x}_{3}\right)$ in $X_{2}$ for which the stable separatrices are, respectively, the $x_{2}$-axis and the $x_{3}$-axis.

If $x_{1} \neq 0, x_{2} \neq 0, x_{3}=0$, the nontrivial nullclines are :

$$
\begin{aligned}
& \mu_{1}\left(s^{\circ}-\sum_{i=1}^{2} \frac{1}{y_{s i}} x_{i}, r^{\circ}-\sum_{i=1}^{2} \frac{1}{y_{r i}} x_{i}, x_{1}\right)-D=0, \\
& \mu_{2}\left(s^{\circ}-\sum_{i=1}^{2} \frac{1}{y_{s i}} x_{i}, r^{\circ}-\sum_{i=1}^{2} \frac{1}{y_{r i}} x_{i}, x_{2}\right)-D=0 .
\end{aligned}
$$

In the proof of Theorem 3.4 we showed that the model (7) has a unique positive equilibrium which is globally asymptotically stable. Hence, the system (10) has an equilibrium point $E_{12}\left(\bar{x}_{1}, \bar{x}_{2}, 0\right)$ in $X_{2}$, at which the jacobian matrix is :

$$
J\left(E_{12}\right)=\left[\begin{array}{ccc}
\bar{x}_{1} \bar{a}_{1} & \bar{x}_{1} \bar{b}_{12} & \bar{x}_{1} \bar{b}_{13} \\
\bar{x}_{2} \bar{b}_{21} & \bar{x}_{2} \bar{a}_{2} & \bar{x}_{2} \bar{b}_{23} \\
0 & 0 & \bar{\mu}_{3}-D
\end{array}\right],
$$

where :

$$
\begin{aligned}
& \bar{\mu}_{j}:=\mu_{j}\left(s^{\circ}-\sum_{i=1}^{2} \frac{1}{y_{s i}} \bar{x}_{i}, r^{\circ}-\sum_{i=1}^{2} \frac{1}{y_{r i}} \bar{x}_{i}, \bar{x}_{j}\right), \\
& \bar{a}_{j}:=-\frac{1}{y_{s j}} \frac{\partial p_{j}}{\partial s}\left(s^{\circ}-\sum_{i=1}^{2} \frac{1}{y_{s i}} \bar{x}_{i}, \bar{x}_{j}\right)+\frac{\partial p_{j}}{\partial x_{j}}\left(s^{\circ}-\sum_{i=1}^{2} \frac{1}{y_{s i}} \bar{x}_{i}, \bar{x}_{j}\right) \text { or } \\
& \bar{a}_{j}:=-\frac{1}{y_{r j}} \frac{\partial q_{j}}{\partial r}\left(r^{\circ}-\sum_{i=1}^{2} \frac{1}{y_{s i}} \bar{x}_{i}, \bar{x}_{j}\right)+\frac{\partial q_{j}}{\partial x_{j}}\left(r^{\circ}-\sum_{i=1}^{2} \frac{1}{y_{s i}} \bar{x}_{i}, \bar{x}_{j}\right), \\
& \bar{b}_{j k}:=-\frac{1}{y_{s k}} \frac{\partial p_{j}}{\partial s}\left(s^{\circ}-\sum_{i=1}^{2} \frac{1}{y_{s i}} \bar{x}_{i}, \bar{x}_{j}\right) \text { or } \\
& \bar{b}_{j k}:=-\frac{1}{y_{r k}} \frac{\partial q_{j}}{\partial r}\left(r^{\circ}-\sum_{i=1}^{2} \frac{1}{y_{s i}} \bar{x}_{i}, \bar{x}_{j}\right) \text { with } k \in\{1,2,3\}, k \neq j .
\end{aligned}
$$


The characteristic equation of $J\left(E_{12}\right)$ is given by :

$$
\left(\bar{\mu}_{3}-D-v\right)\left(v^{2}+A v+B\right)=0,
$$

where

$$
\begin{aligned}
& A:=-\bar{x}_{1} \bar{a}_{1}-\bar{x}_{2} \bar{a}_{2}, \\
& B:=\bar{x}_{1} \bar{x}_{2}\left(\bar{a}_{1} \bar{a}_{2}-\bar{b}_{12} \bar{b}_{21}\right) .
\end{aligned}
$$

One has :

$$
\begin{aligned}
\bar{a}_{1} \bar{a}_{2}-\bar{b}_{12} \bar{b}_{21}= & \frac{1}{y_{s 1} y_{s 2}} \frac{\partial p_{1}}{\partial s} \frac{\partial p_{2}}{\partial s}+\frac{\partial p_{1}}{\partial x_{1}} \frac{\partial p_{2}}{\partial x_{2}}-\frac{1}{y_{s}} \frac{\partial p_{1}}{\partial s} \frac{\partial p_{2}}{\partial x_{2}} \\
& -\frac{1}{y_{s}} \frac{\partial p_{2}}{\partial s} \frac{\partial p_{1}}{\partial x_{1}}-\frac{1}{y_{s} 2} \frac{\partial p_{1}}{\partial s} \frac{1}{y_{s} 1} \frac{\partial p_{2}}{\partial s}>0
\end{aligned}
$$

or

$$
\begin{aligned}
\bar{a}_{1} \bar{a}_{2}-\bar{b}_{12} \bar{b}_{21}= & \frac{1}{y_{r 1} y_{r 2}} \frac{\partial q_{1}}{\partial r} \frac{\partial q_{2}}{\partial r}+\frac{\partial q_{1}}{\partial x_{1}} \frac{\partial q_{2}}{\partial x_{2}}-\frac{1}{y_{r 1}} \frac{\partial q_{1}}{\partial r} \frac{\partial q_{2}}{\partial x_{2}} \\
& -\frac{1}{y_{r 2}} \frac{\partial q_{2}}{\partial r} \frac{\partial q_{1}}{\partial x_{1}}-\frac{1}{y_{r 2}} \frac{\partial q_{1}}{\partial r} \frac{1}{y_{r 1}} \frac{\partial q_{2}}{\partial r}>0 .
\end{aligned}
$$

Hence, the coefficients $A$ and $B$ are positive. According to Routh Criterion (see for example [18]), the equation $v^{2}+A v+B=0$ has two eigenvalues with negative real parts. The persistence result of Theorem 2.5 implies that the other eigenvalue $\bar{\mu}_{3}-D$ must be positive. Hence, $E_{12}$ is a saddle point in $X_{2}$. The stable manifold is the $x_{1} x_{2}$-plane.

If $x_{1} \neq 0, x_{3} \neq 0, x_{2}=0$, or $x_{2} \neq 0, x_{3} \neq 0, x_{1}=0$, one obtains two other saddle points $E_{13}$ and $E_{23}$ in $X_{2}$ the stable manifolds of which are, respectively, the $x_{1} x_{3}$-plane and the $x_{2} x_{3}$-plane.

3. Thieme-Zhao's conditions: Now, having the set of the equilibria of the system (10) in $X$, namely

$$
M=\left\{E_{0}, E_{1}, E_{2}, E_{3}, E_{12}, E_{13}, E_{23}\right\},
$$

we begin to show that the three hypotheses of Thieme-Zhao's theorem ([19,22], see also the Appendix) are verified.

Condition $T 1$ :If $\left(x_{1}(0), x_{2}(0), x_{3}(0)\right) \neq E_{0} \in M$, we distinguish the following cases :

If $x_{1}(0) \neq 0, x_{2}(0)=x_{3}(0)=0$, since $x_{i}(t) \equiv 0$ for $i \in\{2,3\}$, the system (10) simply becomes :

$$
\dot{x}_{1}=\left(\mu_{1}\left(s^{\circ}-\frac{1}{y_{s 1}} x_{1}, r^{\circ}-\frac{1}{y_{r 1}} x_{1}, x_{1}\right)-D\right) x_{1} .
$$

It admits two equilibria, namely the origin which is clearly unstable and $\tilde{x}_{1}$ in $] 0, \min \left(y_{s} s^{\circ}\right.$, $y_{r 1} r^{\circ}$ ) [ for which the global asymptotic stability is obvious according to what precedes and corresponds to the point $E_{1}$. The cases $x_{1}(0)=0, x_{2}(0) \neq 0, x_{3}(0)=0$ and $x_{1}(0)=$ $0, x_{2}(0)=0, x_{3}(0) \neq 0$ are similar. It means that all solutions starting in $X_{2}$ stay in $X_{2}$ and converge to $E_{1}, E_{2}$ or $E_{3}$. 
If $x_{1}(0) \neq 0, x_{2}(0) \neq 0, x_{3}(0)=0$, the system (10) becomes :

$$
\begin{aligned}
& \dot{x}_{1}=\left(\mu_{1}\left(s^{\circ}-\sum_{i=1}^{2} \frac{1}{y_{s i}} x_{i}, r^{\circ}-\sum_{i=1}^{2} \frac{1}{y_{r i}} x_{i}, x_{1}\right)-D\right) x_{1}, \\
& \dot{x}_{2}=\left(\mu_{2}\left(s^{\circ}-\sum_{i=1}^{2} \frac{1}{y_{s i}} x_{i}, r^{\circ}-\sum_{i=1}^{2} \frac{1}{y_{r i}} x_{i}, x_{2}\right)-D\right) x_{2} .
\end{aligned}
$$

In the proof of Theorem 3.4, we already showed that this system has a unique positive equilibrium point which is G.A.S. and denoted by $\left(\bar{x}_{1}, \bar{x}_{2}\right)$. Hence, the solutions of (10) such that $x_{1}(0) \neq 0, \quad x_{2}(0) \neq 0, \quad x_{3}(0)=0$ converge to the corresponding equilibria $E_{12}\left(\bar{x}_{1}, \bar{x}_{2}, 0\right) \in M$. The cases $x_{1}(0) \neq 0, x_{3}(0) \neq 0, x_{2}(0)=0$ and $x_{2}(0) \neq 0, x_{3}(0) \neq$ $0, x_{1}(0)=0$ are analogous and the considered solutions converge, respectively, to $E_{13}$ and $E_{23}$.

Condition $T 2$ : The equilibrium points of $M$ are hyperbolic. The origin is unstable, and the others are saddle points. Therefore, the singleton of each point of $M$ is an isolated invariant set. Moreover, the persistence of the system (3) as showed in Sect. 2 means that the set $M$ is a repeller for $X_{1}$.

Condition T3 : Recall that the stable manifolds of $E_{0}, E_{i}(i \in\{1,2,3\})$ and $E_{i j}$ $(i, j \in\{1,2,3\}$ with $i<j)$ are, respectively, $\left\{E_{0}\right\}$, the axes $\left(O x_{i}\right)$ and the planes $P_{i j}:=\left\{\left(x_{i}, x_{j}\right) \in \mathbb{R}_{+}^{2}: x_{i} \neq 0\right.$ and $\left.x_{j} \neq 0\right\}$. Thus, the only chains occurring in $X_{2}$ are :

$$
\begin{aligned}
& E_{0} \rightarrow E_{i}, E_{1} \rightarrow E_{12}, E_{2} \rightarrow E_{23}, E_{3} \rightarrow E_{13}, \\
& E_{0} \rightarrow E_{i j}, E_{1} \rightarrow E_{13}, E_{2} \rightarrow E_{12}, E_{3} \rightarrow E_{23} .
\end{aligned}
$$

There is no cycle (closed chain) in $X_{2}$. In virtue of Theorem [19] and [22], $X_{2}$ is a uniform repeller for $X_{1}$. Actually, $X_{1}$ being a convex set, there exists at least an equilibrium point in $X_{1}$. Hence, the system (10) is uniformly persistent.

\section{Numerical simulations}

We give some examples of a model of two and three microorganisms in competition on two complementary nutrients. First, the growth functions $p_{i}, q_{i}$ are of Beddington-DeAngelis type, then we present a case where the growth functions are of the form $p_{i}(s) \theta_{i}\left(x_{i}\right)$, $q_{i}(s) \theta_{i}\left(x_{i}\right)$, where $p_{i}$ and $q_{i}$ are increasing and $\theta_{i}$ is decreasing ${ }^{1}$. The parameters used in these examples do not necessarily have a biological meaning and the simulations are given just as an illustration of the result.

Simulation 1 Let the right-hand side functions of the model (5) be given by :

$$
\begin{aligned}
& p_{1}\left(s, x_{1}\right)=\frac{4 s}{2 x_{1}+s+2}, \quad q_{1}\left(r, x_{1}\right)=\frac{5 r}{2 x_{1}+r+1}, \\
& p_{2}\left(s, x_{2}\right)=\frac{5 s}{x_{2}+s+2}, \quad q_{2}\left(r, x_{2}\right)=\frac{6 r}{2 x_{2}+r+4},
\end{aligned}
$$

while the parameters are $s^{\circ}=10, r^{\circ}=20, D=1, y_{s 1}=2, y_{s 2}=3, y_{r 1}=$ 1 and $y_{r_{2}}=7$. One can check that the assumptions of the theorem are all satisfied. The numerical simulations have been performed with the use of Maple 18. In

1 This multiplicative form is justified for example by Zwietering at al. [23]. 
Fig. 1 Eight trajectories of Simulation 1 in the space $\left(x_{1}, x_{2}\right)$ with the initial data given in the text

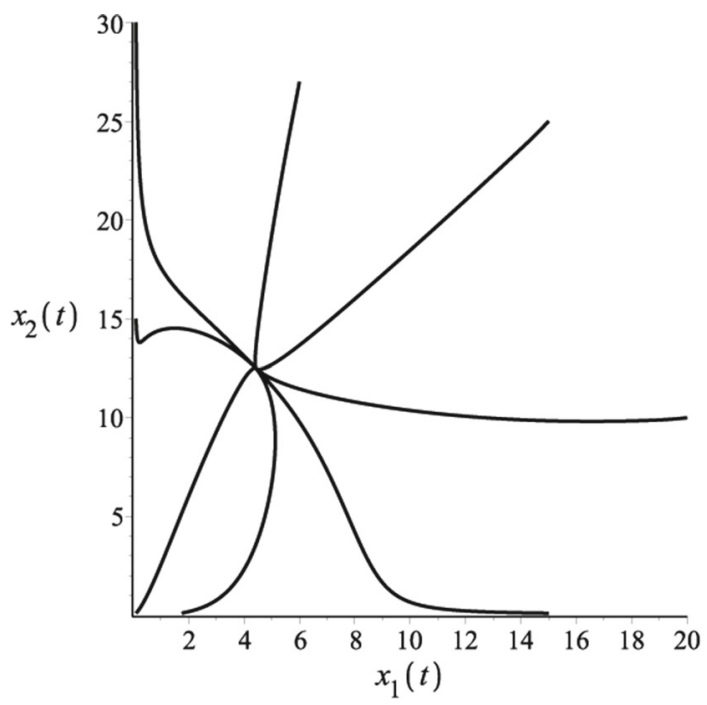

Fig. 1, we represent eight trajectories with initial conditions $\left(s(0), r(0), x_{1}(0), x_{2}(0)\right)=$ $(2.5,3.5,15,25),(2.5,3.5,20,10),(2.5,3.5,0.1,15),(2.5,3.5,6,27),(2.5,3.5,15,0.1)$, $(2.5,3.5,1.75,0.1),(2.5,3.5,0.1,0.1)$ and $(2.5,3.5,0.1,30)$ in the phase space $\left(x_{1}, x_{2}\right)$. These numerical simulations show that the solutions converge to the positive equilibrium point, in accordance with the uniform persistence and the global asymptotic stability property of Theorems 3.3 and 3.4.

Simulation 2 We make the following choice of the functions and the parameters occurring in the model (8)

$$
\begin{aligned}
& p_{1}\left(s, x_{1}\right)=\frac{4 s}{2 x_{1}+s+2}, \quad q_{1}\left(r, x_{1}\right)=\frac{5 r}{2 x_{1}+r+1}, \\
& p_{2}\left(s, x_{2}\right)=\frac{5 s}{x_{2}+s+2}, \quad q_{2}\left(r, x_{2}\right)=\frac{6 r}{2 x_{2}+r+4}, \\
& p_{3}\left(s, x_{3}\right)=\frac{4 s}{x_{3}+s+2}, \quad q_{3}\left(r, x_{3}\right)=\frac{7 r}{x_{3}+r+2},
\end{aligned}
$$

and $s^{\circ}=30, r^{\circ}=35, D=1, y_{s_{1}}=5, y_{s_{2}}=8, y_{s_{3}}=5, y_{r 1}=8, y_{r 2}=$ 4 and $y_{r 3}=9$. Six trajectories are represented in Fig. 2 , namely those with initial conditions $\left(s(0), r(0), x_{1}(0), x_{2}(0), x_{3}(0)\right)=(2.5,3.5,30,85,1),(2.5,3.5,30,5,80)$, $(2.5,3.5,30,30,25),(2.5,3.5,0.5,0.5,0.5),(2.5,3.5,5,30,70)$ and $(2.5,3.5,10,90,30)$. The uniform persistence of the three species of microorganisms is confirmed, and the solutions seem to converge to a positive equilibrium point.

Simulation 3 Now, the right-hand side functions of (5) are given by :

$$
\begin{array}{ll}
p_{1}(s)=\frac{4 s}{2+s}, & q_{1}(r)=\frac{5 r}{2+r}, \quad \theta_{1}\left(x_{1}\right)=\frac{1}{1+1.5 x_{1}}, \\
p_{2}(s)=\frac{5 s}{1+s}, & q_{2}(r)=\frac{6 r}{2+r}, \quad \theta_{2}\left(x_{2}\right)=\frac{1}{1+2 x_{2}},
\end{array}
$$


Fig. 2 Six trajectories of Simulation 2 in the space $\left(x_{1}, x_{2}, x_{3}\right)$ with the initial data given in the text

Fig. 3 Eight trajectories of Simulation 3 in the space $\left(x_{1}, x_{2}\right)$ with the initial data given in the text
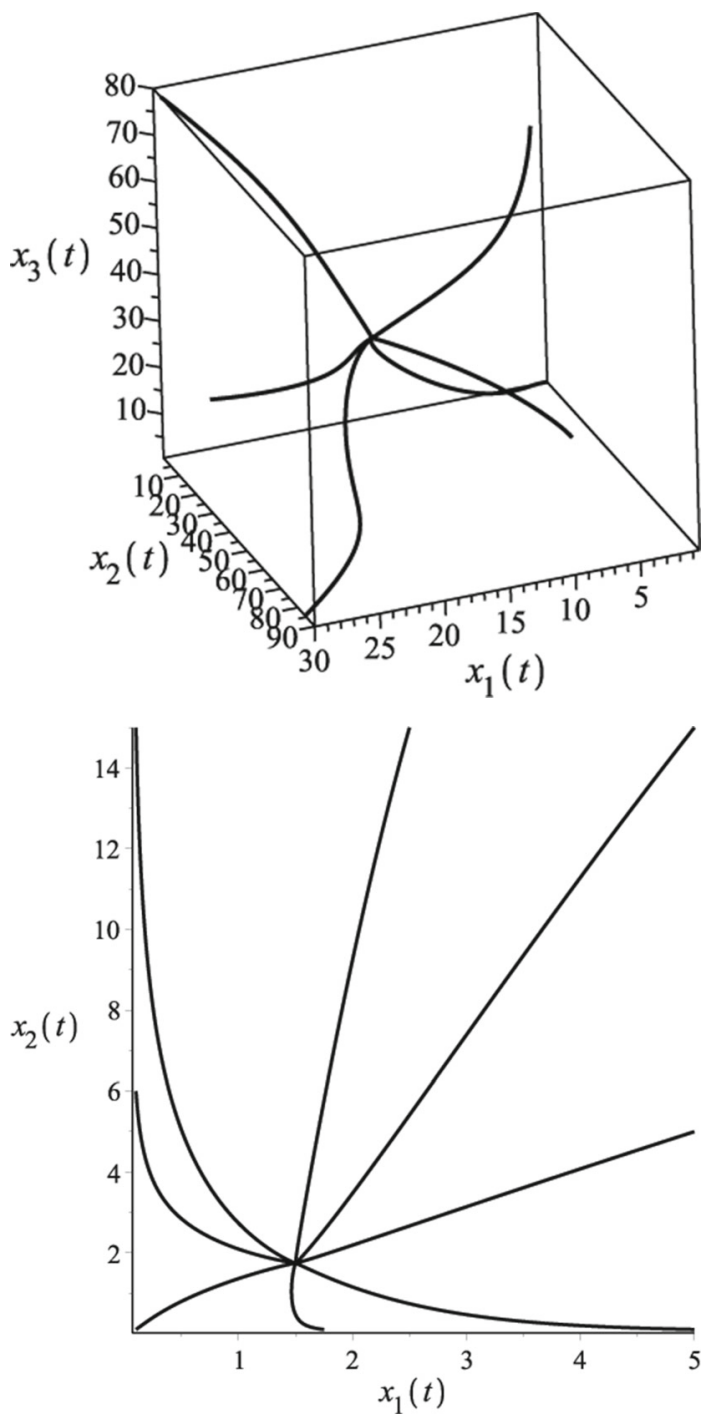

with $s^{\circ}=10, r^{\circ}=20, D=1, y_{s_{1}}=2, y_{s 2}=3, y_{r 1}=1$ and $y_{r_{2}}=7$. In Fig. 3, we represent eight trajectories with initial conditions $\left(s(0), r(0), x_{1}(0), x_{2}(0)\right)=$ $(2.5,3.5,5,5),(2.5,3.5,5,15),(2.5,3.5,0.1,15),(2.5,3.5,2.5,15),(2.5,3.5,5,0.1)$, $(2.5,3.5,1.75,0.1),(2.5,3.5,0.1,0.1)$ and $(2.5,3.5,0.1,6)$ in the phase space $\left(x_{1}, x_{2}\right)$.

Simulation 4 We consider the model (8) with the following functions :

$$
\begin{array}{lll}
p_{1}(s)=\frac{4 s}{2+s}, & q_{1}(r)=\frac{5 r}{2+r}, & \theta_{1}\left(x_{1}\right)=\frac{1}{1+x_{1}}, \\
p_{2}(s)=\frac{5 s}{1+s}, & q_{2}(r)=\frac{6 r}{2+r}, & \theta_{2}\left(x_{2}\right)=\frac{1}{1+x_{2}}, \\
p_{3}(s)=\frac{4 s}{1+s}, & q_{3}(r)=\frac{7 r}{1+r}, & \theta_{3}\left(x_{3}\right)=\frac{1}{1+x_{3}} .
\end{array}
$$


Fig. 4 Six trajectories of Simulation 4 in the space $\left(x_{1}, x_{2}, x_{3}\right)$ with the initial data given in the text

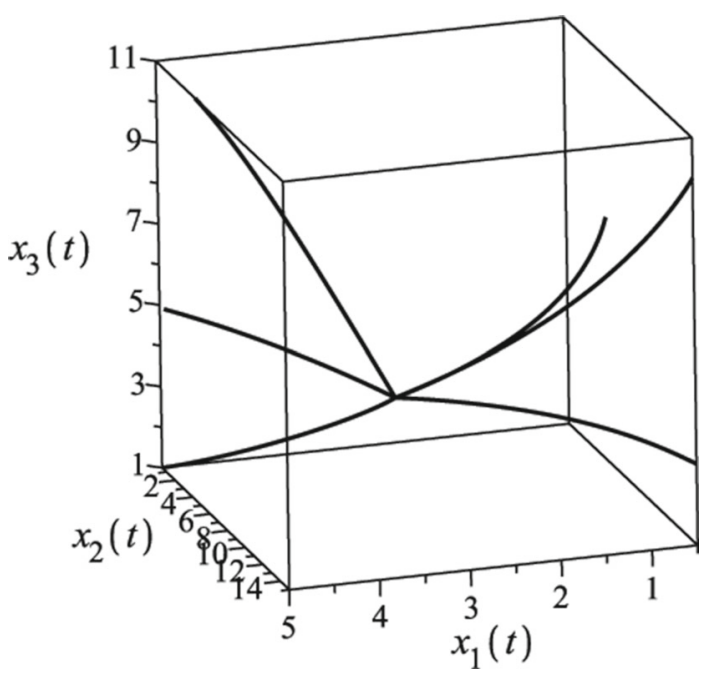

The numerical simulation is performed with the parameters $s^{\circ}=30, r^{\circ}=35, D=1, y_{s_{1}}=$ 5, $y_{s_{2}}=8, y_{s 3}=5, y_{r 1}=8, y_{r 2}=4$ and $y_{r 3}=9$. Six trajectories are represented in Fig. 4 , namely those with initial conditions $\left(s(0), r(0), x_{1}(0), x_{2}(0), x_{3}(0)\right)=(2.5,3.5,5,0.5,1)$, $(2.5,3.5,5,5,11),(2.5,3.5,5,1,5),(2.5,3.5,0.5,15,3),(2.5,3.5,0.5,5,7)$ and $(2.5,3.5$, $0.5,15,10)$.

Acknowledgements The authors would like to thank the anonymous referee for these helpful comments and recommendations.

\section{A Appendix}

Corollary A.1 (Hofbauer-Hutson theorem) [7] Let $X \subseteq \mathbb{R}_{+}^{n}$ be closed, $F: X \longrightarrow \mathbb{R}^{n}$ be locally Lipschitz and consider the ordinary differential system

$$
\dot{x}=F(x)
$$

Assume that $X$ is positively invariant for the system (12). Let $S$ be a compact set and $P \in$ $C\left(X, \mathbb{R}_{+}\right) \cap C^{1}(X \backslash S, \mathbb{R})$ be such that $P(x)=0 \Leftrightarrow x \in S$. Moreover, suppose that there exist a lower semicontinuous function $\psi: X \longrightarrow \mathbb{R}$, bounded below, and an $\alpha \in[0,1]$ such that :

(i) $\dot{P}(x) \geq[P(x)]^{\alpha} \psi(x) \forall x \in X \backslash S$,

(ii) $\forall x \in \Sigma \sup _{T \geq 0} \int_{0}^{T} \psi(x(t)) d t>0$,

where $\Sigma$ denotes $S$ or, whenever $S$ is positively invariant, $\bar{\Omega}(S)$. Then $S$ is a uniform repeller.

By $\Omega(S)$ we denote the whole $\omega$-limit of the orbits on $S$.

Theorem A.2 (Thieme-Zhao) [19,22] Let $X \subseteq \mathbb{R}_{+}^{n}$ be closed, $F: X \longrightarrow \mathbb{R}^{n}$ be locally Lipschitz and consider the ordinary differential system

$$
\dot{x}=F(x)
$$


Assume that $X$ is positively invariant for the system (12) which is supposed to be dissipative on $X$. Let $X=X_{1} \cup X_{2}, X_{1} \cap X_{2}=\varnothing$, with $X_{2}$ being a closed subset of $\mathbb{R}^{n}$ and $X_{1}$ positively invariant. Let $M$ be a finite set of equilibria of (12) in $X_{2}$. Assume that :

T1. Every solution that starts in $X_{2}$ and stays in $X_{2}$ for all forward times converges to one of the equilibria in $M$,

T2. Every equilibrium in $M$ is an isolated invariant set in $X$ and a weak repeller for $X_{1}$,

T3. $M$ is acyclic in $X_{2}$.

Then $X_{2}$ is a uniform strong repeller for $X_{1}$. Moreover, if $X_{1}$ is convex, there exists at least one equilibrium point in $X_{1}$.

\section{References}

1. Ajbar, A., Alhumaizi, K.: Dynamics of the Chemostat : A Bifurcation Theory Approach. Taylor and Francis, CRC Press (2011)

2. Arditi, R., Ginzburg, L.: Coupling in predator-prey dynamics: ratio-dependence. J. Theor. Biol. 139, 311-326 (1989)

3. Arditi, R., Ginzburg, L.: How Species Interact : Altering the Standard View on Thophic Ecology. Oxford University Press, Oxford (2012)

4. Borsali, F., Yadi, K.: Contribution to the study of the effect of the interspecificity on a two nutrients competition model. Int. J. Biomath. 8(1), 243-253 (2015)

5. Butler, G.J., Wolkowicz, G.S.K.: Exploitative competition in a chemostat for two complementary, and possible inhibitory, resources. Math. Biosci. 83, 1-48 (1987)

6. Covich, A.: Ecological economics of foraging among coelving animals and plants. Ann. Mo. Bot. Gard. 61, 794-805 (1974)

7. Fonda, A.: Uniformly persistent semidynamical systems. Proc. Am. Math. Soc. 104, 111-116 (1988)

8. Harmand, J., Lobry, C., Rapaport, A., Sari, T.: The Chemostat : Mathematical Theory of Microorganism Cultures, Chemical Engineering Series. Wiley-ISTE, UK (2017)

9. Hofbauer, J.: A general cooperation theorem for hypercycles. Monatsh. Math. 91, 233-240 (1981)

10. Hsu, S.B., Cheng, K.S., Hubbell, S.P.: Exploitative competition of microorganism for two complementary nutrients in continuous culture. SIAM J. Appl. Math. 41, 422-444 (1981)

11. Hutson, V.: A theorem on average Liapunov functions. Monatsh. Math. 98, 267-273 (1984)

12. Leon, J.A., Tumpson, D.B.: Competition between two species for two complementary or substitutable resources. J. Theor. Biol. 50, 185-201 (1975)

13. Li, B.: Global asymptotic behavior of the chemostat: general response functions and different removal rates. SIAM J. Appl. Math. 59, 411-422 (1999)

14. Li, Z., Xu, R.: Stability analysis of a ratio-dependent chemostat model with time delay and variable yield. Int. J. Biomath. 3(2), 243-253 (2010)

15. Lobry, C., Mazenc, F.: Effect on persistence of intra-specific competition in competition models. Electron. J. Differ. Equ. 2007(125), 1-10 (2007)

16. Mazenc, F., Malisoff, M.: On stability and stabilization for models of chemostats with multiple limiting substrates. J. Biol. Dyn. 6(2), 612-627 (2012)

17. Rapport, D.J.: An optimization model of food selection. Ann. Nat. 105, 575-587 (1971)

18. Smith, H.L., Waltman, P.: The Theory of the Chemostat. Cambridge University Press, Cambridge (1995)

19. Thieme, H.R.: Persistence under relaxed point-dissipativity (with application to an epidemic model). SIAM J. Math. Anal. 24, 407-435 (1993)

20. Tilman, D.: Resource Competition and Community Structure. Princeton University Press, Princeton (1982)

21. Vidyasagar, M.: Decomposition techniques for large-scale systems with nonadditive interactions: stability and stabilizability. IEEE Trans. Automat. Control 25, 773-779 (1980)

22. Zhao, X.-Q.: Dynamical Systems in Population Biology, CMS Books in Mathematics. Springer, New York (2003)

23. Zwietering, M.H., Wijtes, T., De Wit, J.C., van't Riet, K.: A decision support system for prediction of the microbial spoilage in foods. J. Food Prot. 55, 973-979 (1992) 